\title{
WiMAX Mesh Topology with Modified Fair Centralized Scheduling and Routing Algorithms Based on Testing Feature of Down-Node Group
}

\author{
Z. K. Farej Dr. A. I. A. Jabbar \\ Elect. Eng. Dept. / University of Mosul
}

\begin{abstract}
Multiple access interferences are the major limiting factors for the performance of multihop WiMAX based Mesh Network (WMN). The routing algorithms play an important role in reducing such interferences among the nodes of the WMN. In this paper a new Routing Tree Construction Algorithm (RTCA) which is based on Balancing the data and Parallelizing the routes of the data from nodes to $\mathrm{BS}$ is proposed and called BalancedParalleled RTCA (Bala-Para-RTCA). This algorithm has a new Down-Node Group Testing (DNGT) feature. This feature increases the conversion of primary into secondary interferences which (in conjunction with directivity) can be avoided significantly by the Modified Fair Relay Centralized Scheduling (MFRCS) algorithm, as a result concurrent transmissions over a certain slot will be increased. So system performance is enhanced in terms of scheduling length, links concurrency ratio (LCR) and average transmission delay (ATD). Without directivity and relative to the standard Breadth First Tree (BFT) RTCA, the simulation results of the proposed RTCA show concurrency ratio enhancement of $15.38 \%$ and $19.4 \%$ without and with DNGT respectively.
\end{abstract}

Keywords: WMN, Bala-Para-RTCA, DNGT, MFCS, LCR, Schedule Length

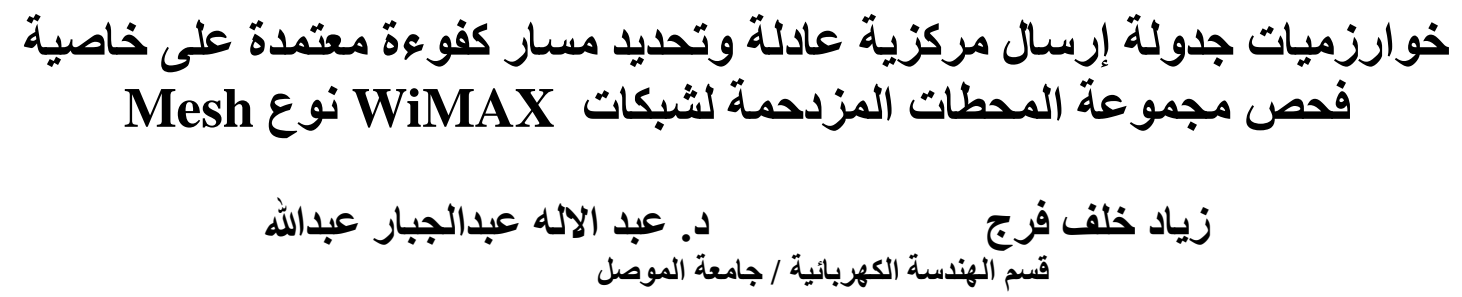

الخلاصة

إن التاخلات الناتجة من تعدد الوصول (Multiple Access) تعتبر العامل الرئيسي الذي يحد من أداء شبكات الـ (WiMAX)

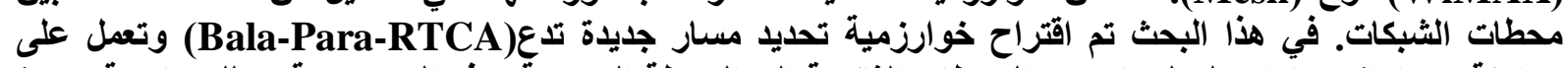

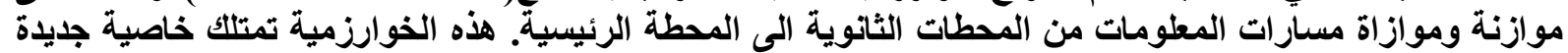

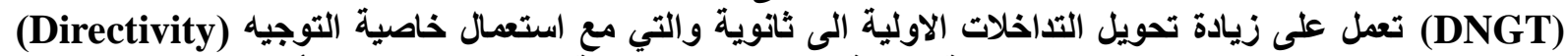

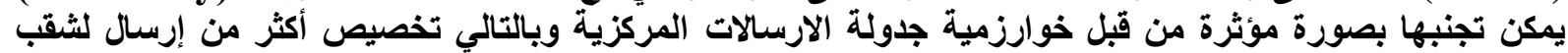

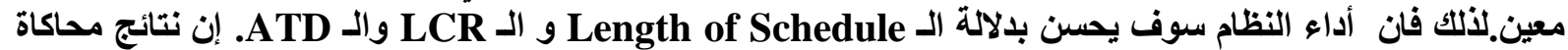

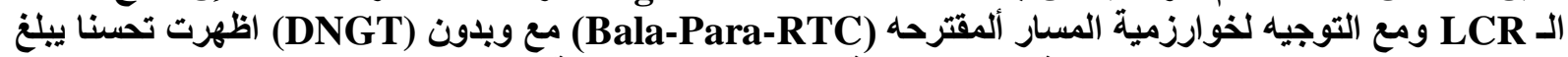

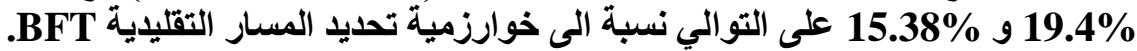




\section{Introduction}

WiMax (world interoperability for microwave access) is a family of IEEE 802.16 standards with the aim of providing high-speed wireless access to a large number of users in the metropolitan area network (MAN). WiMAX networks operate synchronously in a time slotted mode and can be configured to work in different modes, point-to-multipoint (PMP) or Mesh mode[1].

In PMP, the connection is direct between each Subscriber Station (SS) and Base Station (BS) through single hop where as in mesh mode, the BS and SSs coordinate among themselves to transmit packets in a multi-hop environment, and all nodes share the channel in a time division multiple access (TDMA) fashion.

It is also necessary to allocate time slots without collision over the network to achieve the assigned bandwidth for each connection.

The scheduling and RTCAs are important issues in WiMAX networks, because the IEEE 802.16 mesh mode has defined the messages and signalling mechanisms for transmission scheduling, but the minislots which are assigned to the different stations are left unspecified, therefore an efficient channel (minislots) allocation algorithm which allows more concurrent transmission among links over the same timeslot is needed. Accordingly and in order to allow more concurrent transmission, the interferences among the transmission links should be reduced.

\section{Related work}

Scheduling and route construction problems are investigated and different strategies are used to reduce the interference in the routing tree. For example in [2], two tree construction algorithms: min max degree and maximum parallelism are proposed. The first one aims to reduce the maximal node degree in a routing tree while the latter one can maximize the number of link pairs that can work simultaneously without interference. In [3] an interferenceaware routing tree is constructed by creating a routing path between each SS and the BS that has the minimum degree of interference effect. The authors in [4] proposed a collision-free centralized scheduling where a SS is assigned service tokens based on its traffic demand. In [5] a general algorithm for SSs is designed to achieve concurrent transmissions, it enhances the throughput of information streams and gives a method to construct the necessary routing tree of the system. In [6] a construction scheme for the centralized scheduling is proposed to exploit the spatial reuse, the scheme has the capability to take into account both interference and bandwidth request during the construction of a routing tree.

Current research on WiMAX mesh centralized scheduling mainly focuses on minislot and spectrum reuse which are constrained by the routing tree structure algorithm.

In this paper, the proposed Bala-Para-RTCA with its new DNGT feature is proposed to reduce or convert primary interference into secondary interference. Consequently, in conjunction with directivity which makes use of spatial reuse efficiently, the modified MFRCS will assign more concurrent transmissions over a certain slot for variable traffic demand. Therefore, the length of schedule will be minimized and the system performance is enhanced.

\section{Background Theory}

\subsection{WiMAX Mesh Mode}

Time Division Multiple Access (TDMA) is the protocol being used for channel access. In WiMAX mesh mode, both centralized and distributed schedulings are supported, in this paper 
focusing will be on centralized scheduling which handles both the uplink and downlink traffics. Time Division Duplex (TDD) is used to share the channel between uplink and downlink. The mesh BS acts as the centralized scheduler and determines the allocation of the minislots among all the SSs. The time period for centralized scheduling is called scheduling period. It has two steps:

1) The SSs send bandwidth requests messages which are routed to the MBS along the routing tree. Each SS not only send its own bandwidth request but also relays that of its children in the scheduling tree. The SSs transmit Mesh Centralized Scheduling (MSH-CSCH) Request messages in such an order that the sponsor nodes always transmit after all their children. In this way, the mesh BS collects bandwidth requests from all the SSs.

2) The mesh BS calculates and distributes the schedule by broadcasting the MSH-SCH Grant message, which is propagated to all the SSs along the routing tree. Since the dominant traffic in a WiMAX mesh network is Internet traffic,

\subsection{Interference Model}

As shown in Fig. (1). The data transmission due to primary and secondary interferences may collide in two ways [4][7][8].

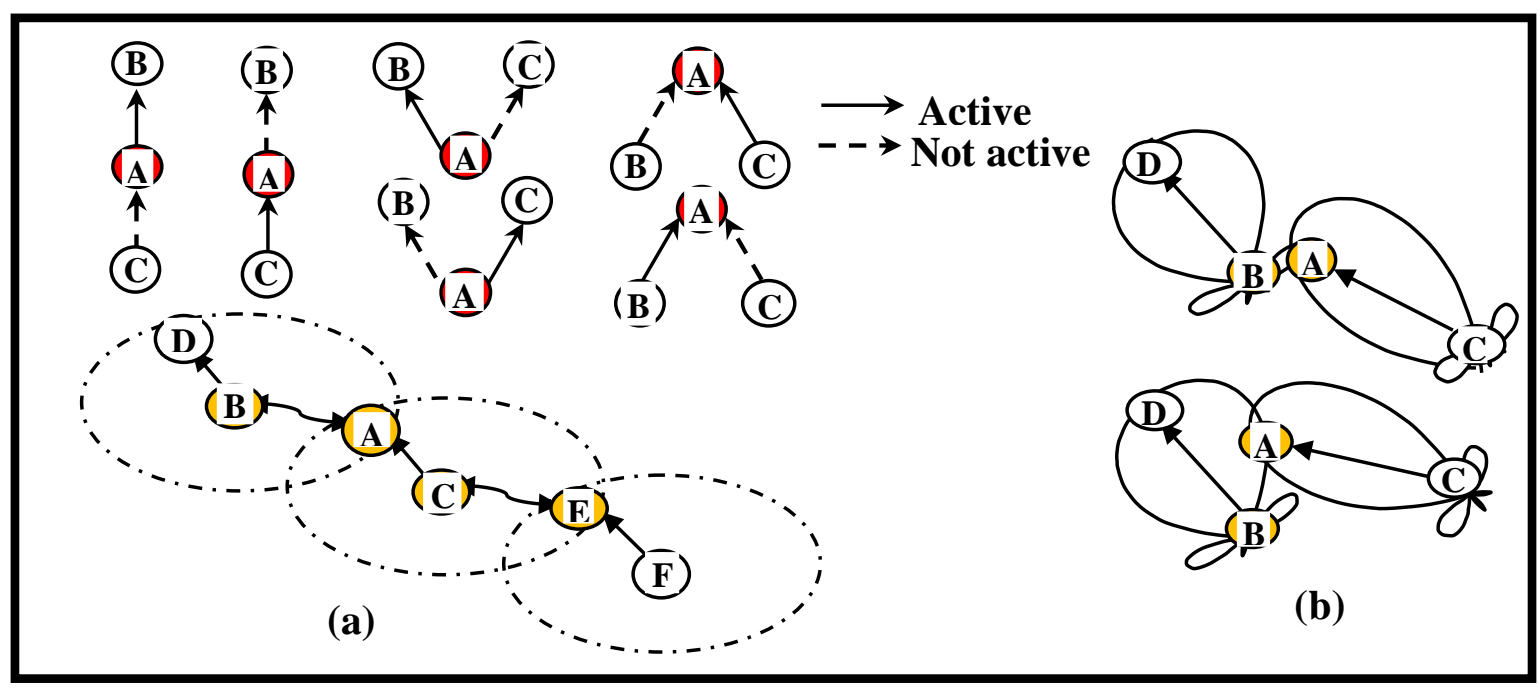

Fig.(1): shows interferences (a) primary and secondary interferences without directivity

(b) places where secondary interferences could happen when directivity introduced.

1. Primary interference (transmission/reception constraint) which occurs when a SS can perform more than one job in a single time slot as follows:

a) The SS cannot transmit and receive simultaneously.

b) The SS cannot transmit/receive more than one packet at the same time.

2. Secondary interference (interference-free constraint ) which occurs when a receiver $R$ tuned to a particular transmitter $\mathrm{T}$ is within the range of another transmitter whose transmission interferes with the transmission of $\mathrm{T}$. 
Fig.(2) illustrates this interference more clearly. The solid lines represent edges in the routing tree and the dashed lines represent interference links. Suppose $\mathrm{SS}_{4}$ transmits to $\mathrm{SS}_{2}$ in certain time slot. Then links $\left(\mathrm{SS}_{2}, \mathrm{BS}\right),\left(\mathrm{SS}_{7}, \mathrm{SS}_{4}\right)$ and $\left(\mathrm{SS}_{8}, \mathrm{SS}_{4}\right)$ are blocked because of primary interference (a) and links $\left(\mathrm{SS}_{5}, \mathrm{SS}_{2}\right)$ and $\left(\mathrm{SS}_{6}, \mathrm{SS}_{2}\right)$ are blocked because of primary interference (b).links $\left(\mathrm{SS}_{1}, \mathrm{BS}\right)$ and $\left(\mathrm{SS}_{3}, \mathrm{BS}\right)$ are blocked due to secondary interference.

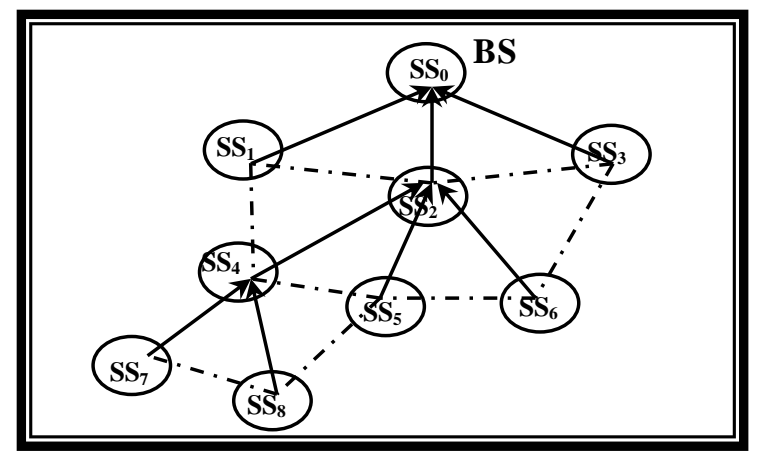

Fig.(2) clarify primary and secondary interferences among nodes of the mesh topology

\section{Features of the Proposed Routing Algorithm}

Fundamentally, the routing algorithm constructs routing tree between SSs and BS. When tree is constructed, according to the proposed routing algorithm, the aim is to convert the primary interferences(b) into secondary, so that it is possible to make use of directivity efficiently in minimizing the secondary interferences among the mesh network links. This can be achieved by applying :

1) Parallelism, it is used to minimize the maximal node degree of the routing tree by examining down-node group (degree) values. Refer to Figure (3a) where $\mathrm{SS}_{1}, \mathrm{SS}_{2}$, and $\mathrm{SS}_{3}$ are at level 1 and $\mathrm{SS}_{4}, \mathrm{SS}_{5}$ and $\mathrm{SS}_{6}$ are at level 2. The down-node groups of $\mathrm{SS}_{1}, \mathrm{SS}_{2}$, and $\mathrm{SS}_{3}$ are $\left(\mathrm{SS}_{4}\right),\left(\mathrm{SS}_{4}, \mathrm{SS}_{5}\right.$ and $\left.\mathrm{SS}_{6}\right)$ and $\left(\mathrm{SS}_{6}\right)$ respectively. The values of these groups are 1,3 and 1 respectively.

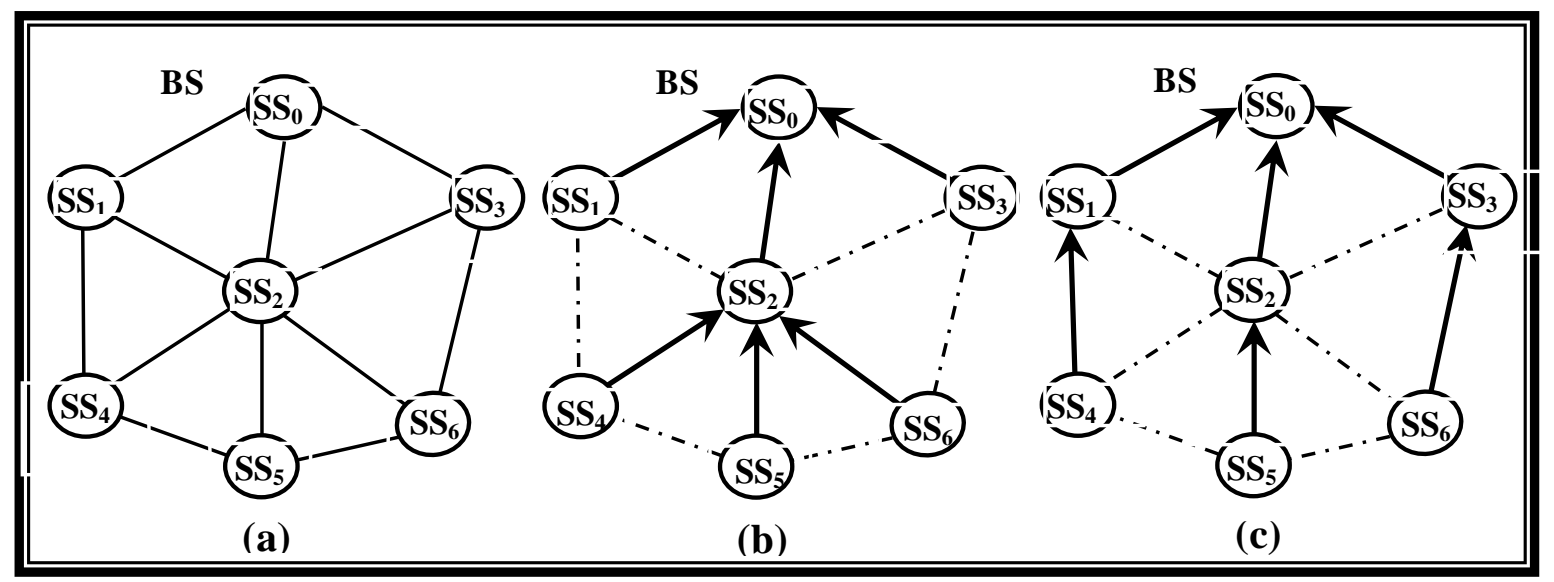

Fig. (3) illustrates the parallelism property of the proposed routing algorithms (a) simple mesh network (b) routing tree without down-node test (c) with down-node test

Without examining down-node group value, possible uplink traffic tree is given in Figure(3b), while when down-node test is applied, the routing tree according to the proposed routing algorithms will be as shown in Figure (3c). Therefore, the maximal degree (primary interference $b$ ) of node $\mathrm{SS}_{2}$ is reduced from 3 to 1 .

2) Balancing the traffic from child nodes to the parent node by examining child bandwidth requests. Figure (4a) shows a simple mesh network with granted BW for each node in PDU with 1 packet size each. 


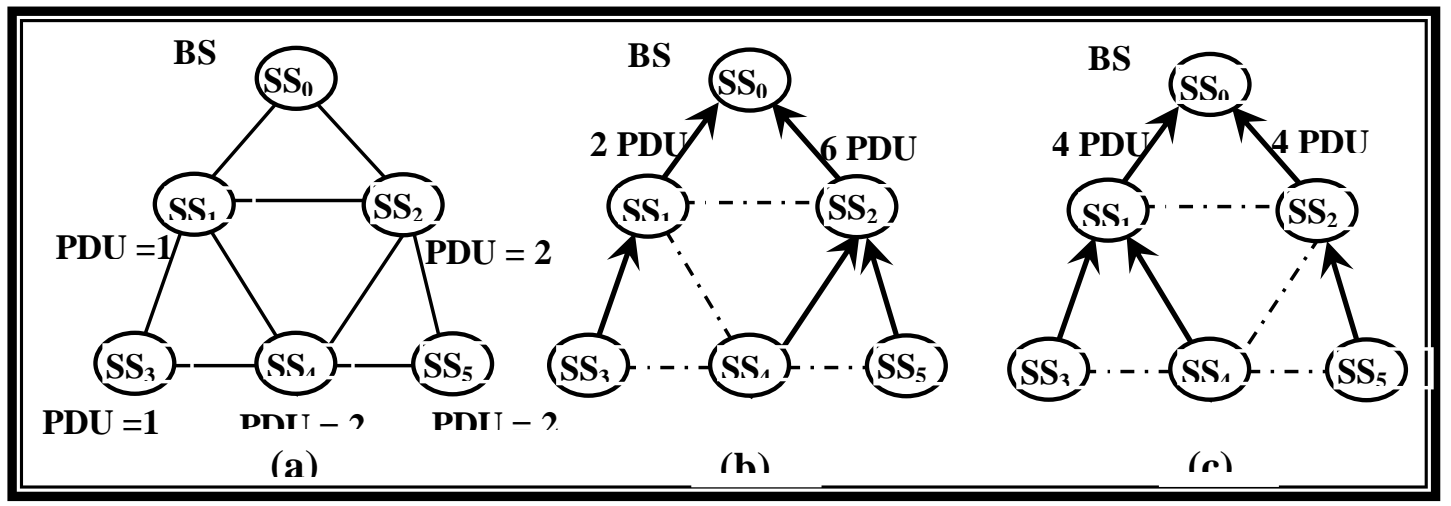

Fig. (4) illustrates the balance property of the proposed routing algorithms (a) simple mesh network (b) routing tree without child BW requests test (c) with child BW requests test

Possible uplink traffic tree constructed without child BW request test is shown in Figure (4b), where the traffics over links $\mathrm{SS}_{1} \rightarrow \mathrm{SS}_{0}$ and $\mathrm{SS}_{2} \rightarrow \mathrm{SS}_{0}$ are not balanced. Figure (4c) shows the balanced traffic over the links (4 packets on each one) when the tree constructed with child BW requests test according to the proposed routing algorithms.

\section{Design of Proposed Routing and Scheduling Algorithms}

\subsection{Routing Tree Construction Algorithm}

The proposed Bala-Para RTCA has the DNGT feature which gives priority for nodes (with highest BW request as primary key and identification ID as secondary key) to choose their routes first. These nodes are members of the down node groups with values greater than two. This feature increases the chance of minimizing the maximal node degree, support quality of service, then increasing the chance of making the system routes of data more parallel (and as a consequence the directivity or spatial reuse will reduce the secondary interferences).

The design according to the proposed routing algorithms should include some network preparation and nodes relationships as follows:

To keep the shortest route paths to the tree root (base station), the topology $G=(V, E)$ of the mesh network is translated into a layer graph $\mathrm{G}_{\mathrm{L}}=(\mathrm{V}, \mathrm{E})$. Each node $\left(S S_{i}\right)$ in $\mathrm{G}_{\mathrm{L}}$ is associated with a layer number $l\left(S S_{i}\right)$ which is equal to the minimal hop count of this node to $S S_{0}$ (BS). Nodes with the same layer number are placed on the same level (see Fig. 5).

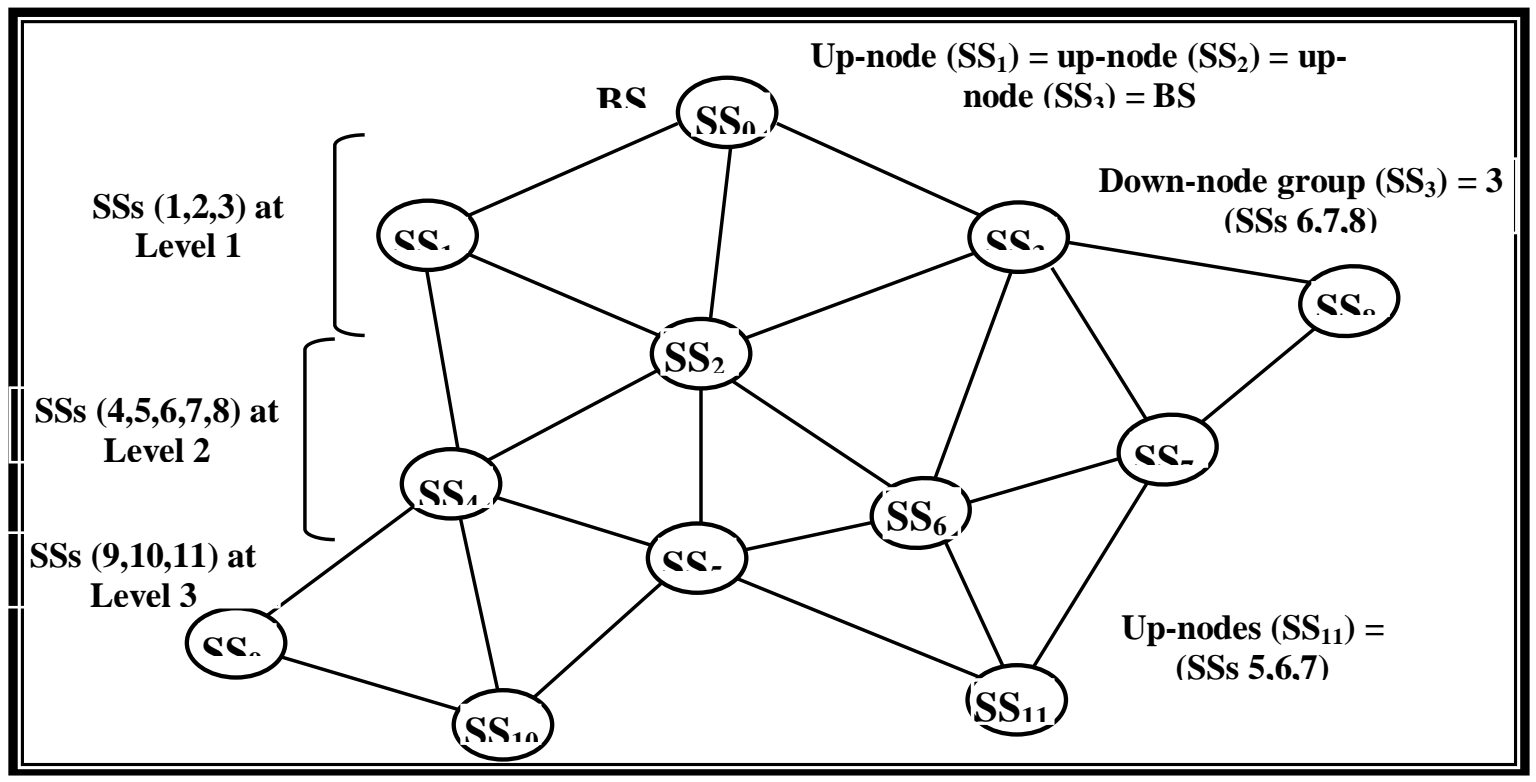

Fig. (5) shows lavered graph $\mathrm{G}_{\mathrm{I}}$ and some nodes relationship in general mesh topologv 
The tree construction flow chart is shown in Fig.6.

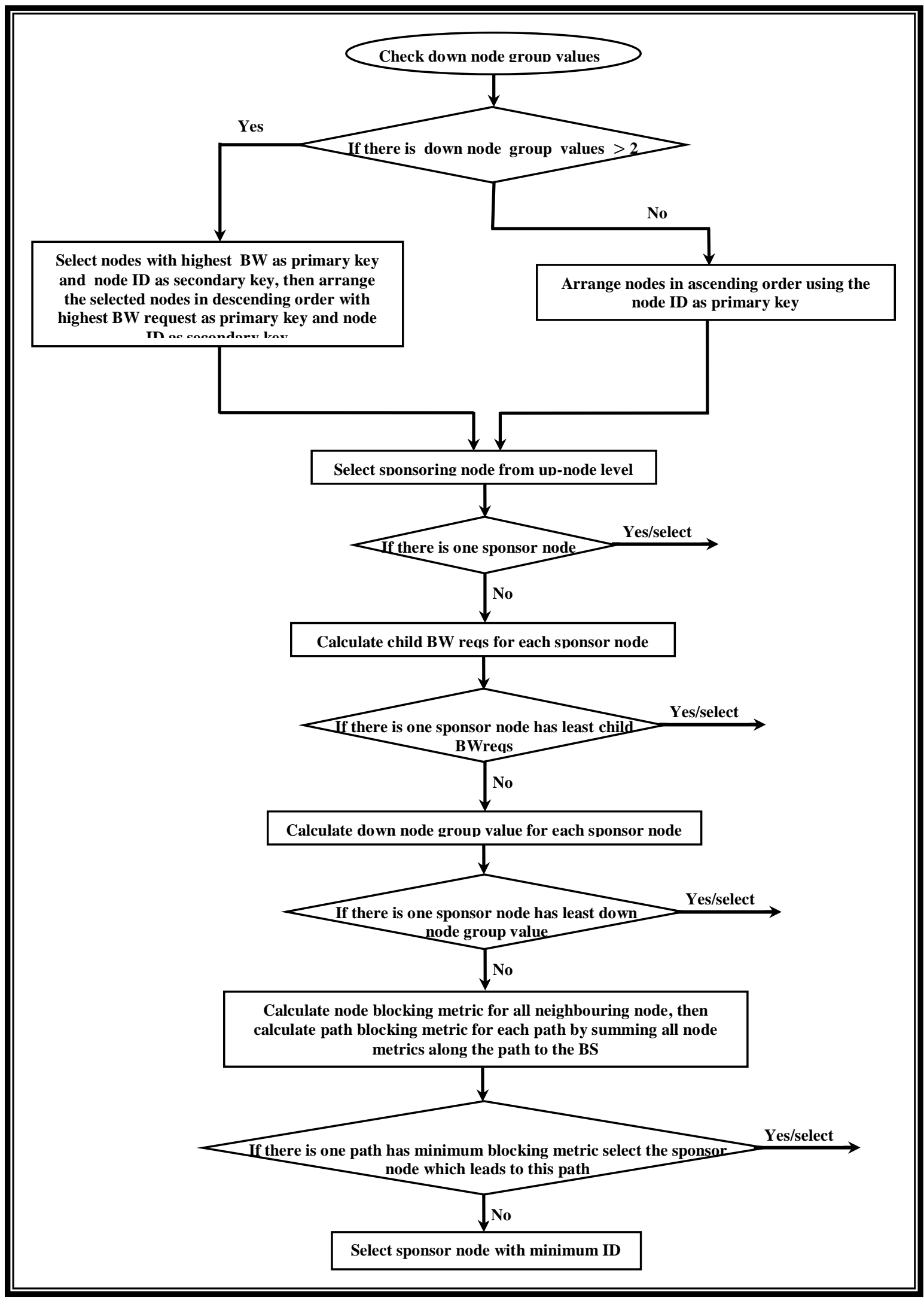

Fig. (6) Flow chart of the proposed Bala-Para routing tree construction algorithm 
The nodes, which are within the Transmission Range (TR) of the BS, join the network in the first layer. The minimum identity will be given to the first node join the network while the last node which joins the network will be given the maximum ID in the last layer (maximum Hop Range (HR) parameter typically five) defined by network descriptor

In Fig.(7), the mesh network in dashed lines with its routing tree in solid lines is constructed by using the breadth-first traversal (BFT) according to the IEEE802.16 standard. When a new node join the network, it must select the shortest path to the base station with the condition that if there are more than one node in the same level then priority will be given to node with minimum ID to choose its path first. This algorithm is suggested by [4] and adopted in this paper for comparison purposes.

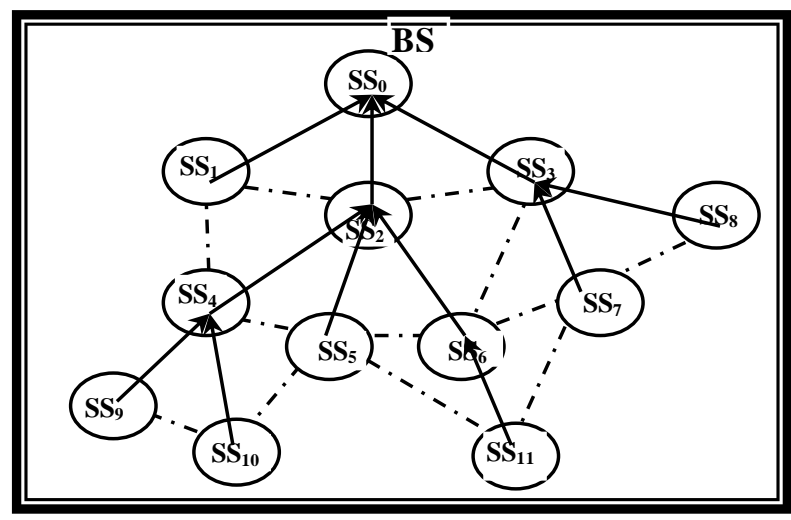

Fig.(7) Shows Routing Tree Construction According to BFT-RTC Algorithm

According to the proposed Bala-Para-RTC algorithm without DNGT feature, Fig.(8) shows the final uplink traffic constructed tree links in solid lines. For simplicity the granted BW for each node is given in the PDU. All nodes are assumed to have same number \& size of PDU . In this algorithm, routs between the nodes and the base station are chosen by given precedence to nodes joining the net first. So the node sequence to be examined is $1,2,3,4,5,6,7,8,9,10$ and 11 . While with DNGT feature the precedence will be given to the following node sequence priority $8,6,3,1,2,4,5,7,9,10$ and 11 . For each node in the up-node set , the child BW reqs, down node degree values and blocking metric values are computed. Then for each node, a parent node is selected from its up-node set and if more than one node exist in its up-node set then the following rules are used to select the proper one between them:

1) Select node with the minimal child BW reqs value.

2) Select node with the minimal down node group degree value.

3) Select node which leads to the minimum path blocking metric.

4) Select node with minimum ID.

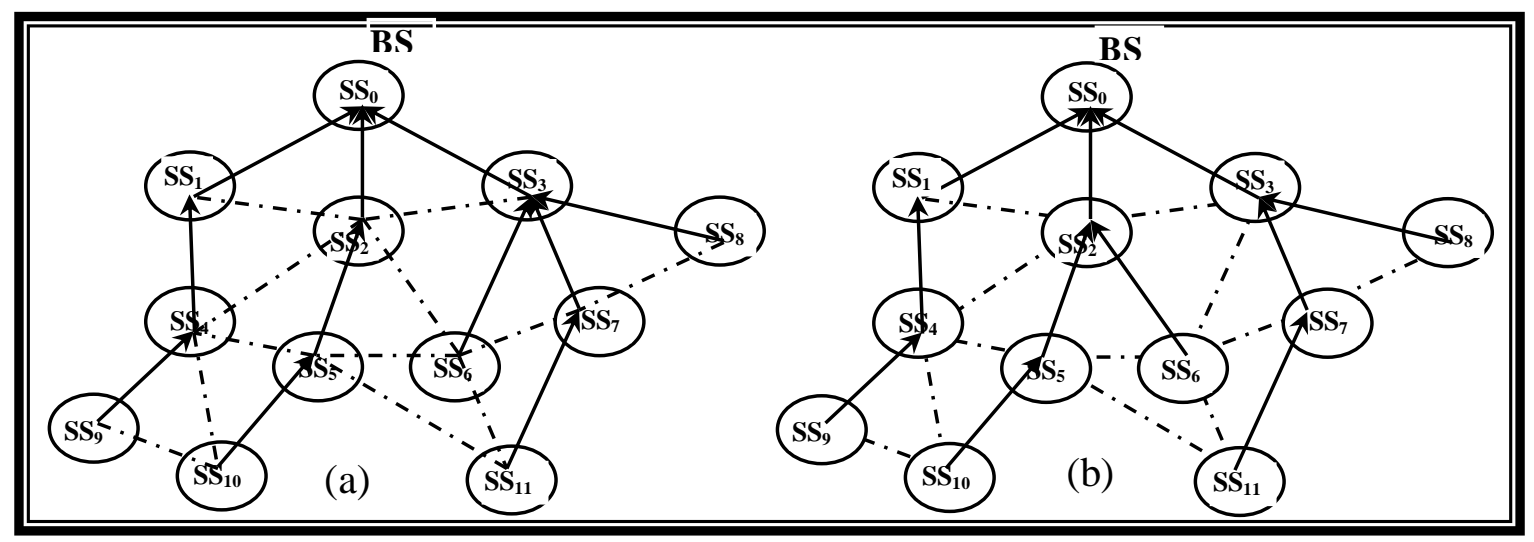

Fig.(8) Routing Tree Constructed According to the proposed Bala-Para-RTC algorithm (a) without (b) with DNGT feature 


\subsection{Proposed Modified Fair Relayed Centralized Scheduling Algorithm}

The principal of scheduling is to find a valid minisolt allocation with minimum $\sum_{\forall S S_{i} \in V} S_{i}$ (accumulated number of slots $S_{i}$ of all active SS in the topology) value which can be achieved by maximizing the degree of minislot reuse.

In the proposed CS algorithm, the links between stations are scheduled in such a way that the transmission of a station can be received collision-free by a given neighbour SS.

The objective of the proposed TDMA CS algorithm is to eliminate the primary and secondary conflicts and to optimize the number of slots in a frame such that minimum latency can be obtained.

In order to design a centralized scheduling algorithm according to the IEEE 802.16 the following assumptions were made:

1. The topology is fixed during the scheduling period.

2. No node can transmit and receive data simultaneously.

3. Any node can not send or receive data if it is within the range of communication between any other two active (scheduled for transmission) nodes.

4. The signal of a node can only cover the range of a single-hope neighborhood.

5. The control and scheduling sub-frame are long enough.

6. Node can transmit one packet in each time slot.

7. The buffer in each node is of the type first in first out (FIFO).

8. Each node generates random numder of packets(one packets for CBR and random number from 0 to 3 for VBR).

9. Node can select suitable transmission power to reach its immediate "receiving" next hope.

The function of the BS is to collect the active SS's bandwidth requests through MSH-CSCH request messages,then it updates the network configuration tree according to the RTC algorithims and propagates the routing tree to active SS through MSH-CSCF message. BS centrelly performs time slot allocation for SSs (according to their BW demands) taking into account the interferences of each SS on its neighboring SSs, as well as avoiding the collsions that may arise from hidden terminals. The assigned time slots to the granted connection will be propagated by the BS to all SSs through MSH-CSCH grant message.

According to the centrelized TDMA scheduling algorithm and in order to achieve high system LCR and provide fairness, the Relay Model CS adapted in this paper gives praiority to the nearer nodes to the BS to transmit first. The algorithm also consider interferences, BW, slot reuse, and QoS.

In this algorithm, link scheduling is used and a SS is assigned service token ST which is based on its traffic demand, this can be performed by intially setting a counter /timer equal to the number of packets or slots for each SS link proportional to the traffic demand (1 for $1 \mathrm{Mbps}, 2$ for $2 \mathrm{Mbps}$...etc),thus, the fairness is guaranteed and no nodes will be starved. A link can be scheduled only if the service token number of its transmitter is nonzero. Also more than one link can be scheduled at the same time slot if they are not interfered with each other according to the interference module described before. Each time after a link is assigned a time slot, the service token of the transmiter is decreased by one and that of the receiver is increased by one. Flow chart of the proposed MFRCS is shown in Fig.(9). 


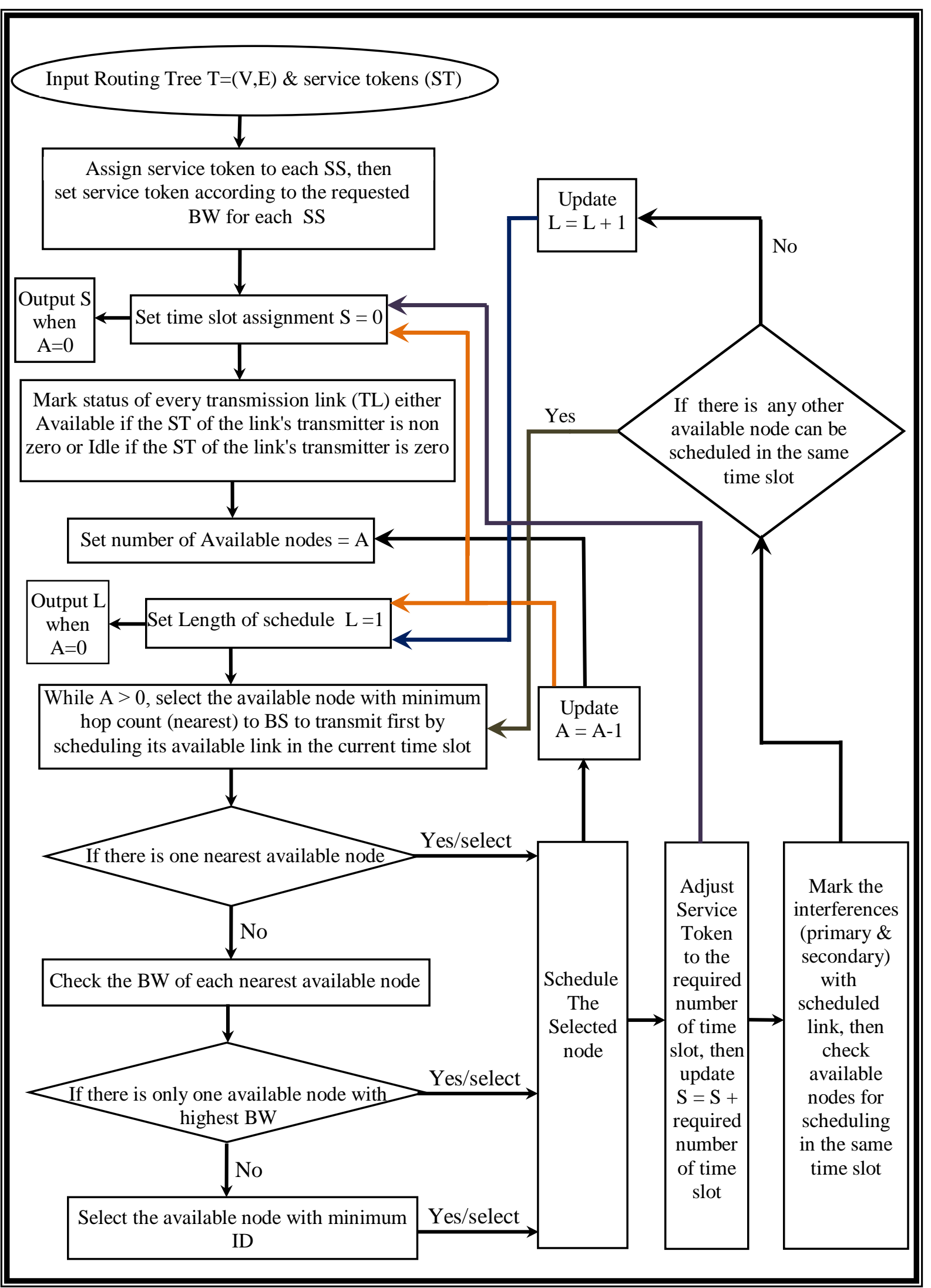

Fig.(9): Flow chart of the proposed scheduling algorithm 


\section{Analytical performance and Results}

The analysis of IEEE 802.16 mesh network is based on the assumption that

1) There is a number of Mesh SS nodes which are randomly scattered

2) A link connectivity graph is based on the assumption that there is a link between two nodes if they are in the range of each other

3) Three routing trees are built using the three construction algorithms summarized in (5).

Assume that the number of SSs in the network is "n", and the uplink and downlink traffic requests are $\mathrm{D}_{\mathrm{u}}(\mathrm{i})$ and $\mathrm{D}_{\mathrm{d}}(\mathrm{i})$ respectively, which have been normalized by $C_{B P S K, 1 / 2}$, ( link capacity with modulation matrix of BPSK 1/2)

If $\quad \mathrm{D}(\mathrm{i})=\mathrm{D}_{\mathrm{u}}(\mathrm{i})+\mathrm{D}_{\mathrm{d}}(\mathrm{i})$

Then the total network demand is $\mathrm{D}$ which is given by

$\mathrm{D}=\sum_{\mathrm{i}=1}^{\mathrm{n}} \mathrm{D}(\mathrm{i})$

Based on the traffic demand of SSs, the uplink $\left(S_{u}(i)\right)$ and downlink $\left(S_{d}(i)\right)$ slot demands of $\mathrm{SS}_{\mathrm{i}}$ can be expressed by the following [10]:

$$
\begin{aligned}
& S_{u}(i)=\left\lceil\frac{D_{u}(i) \cdot T}{R_{u}(i) \cdot \tau}\right\rceil \\
& S_{d}(i)=\left\lceil\frac{D_{d}(i) \cdot T}{R_{d}(i) \cdot \tau}\right\rceil
\end{aligned}
$$

Where: $\quad \mathrm{R}_{\mathrm{u}}(\mathrm{i})$ bits/s and $\mathrm{R}_{\mathrm{d}}(\mathrm{i})$ bits/s, represent the uplink and downlink transmitting rates respectively.

$\mathrm{T}=$ the scheduling period in seconds.

$\tau=$ slot or single transmission opportunity duration in seconds.

To take into account the case where the traffic from a given $\mathrm{SS}_{\mathrm{i}}$. needs to be relayed $\mathrm{h}(\mathrm{i})$ times to reach the destination. let $C(l)$ be the average link capacity in the branch $l$ of the routing tree. $\alpha$ is the weighted factor which varies simultaneously by the active link number and link capacity. Then the actual length of schedule (L) which represent the total number of transmission opportunity (in slot) required to complete the overall transmission can be expressed as [9]:

$$
\mathrm{L}=\sum_{i=1}^{n}(\mathrm{D}(\mathrm{i}) \cdot \mathrm{h}(\mathrm{i})) / \alpha,
$$

where

$$
\alpha=\operatorname{CUR} \bullet \sum_{l=1}^{|\mathrm{E}|} C(l) / C_{B P S K, 1 / 2} \text {. }
$$

$$
\mathrm{h}(\mathrm{i})=\text { the required number of hops for } \mathrm{SS}_{\mathrm{i}} \text { to reach the base station. }
$$

CUR $=\sum_{i=1}^{n} \mathrm{~h}_{i} \mathrm{D}_{i} / \mathrm{nL}[7]$. 
Upper bound of $L=\sum_{i=1}^{n} h_{i} \cdot D_{i}$ when one link scheduled in each slot $(\alpha=1)$.

Lower bound of $L=\sum_{i=1}^{n} D_{i}$ when every SS is one hop $\left(h_{i}=1\right)$ from BS

\subsection{Link Concurrency Ratio (LCR)}

It is used to evaluate the efficiency of the spatial reuse, (i.e. how many links can transmit concurrently at a given time). The LCR can be defined as the ratio of the upper bound scheduling length to the actual scheduling length when spatial reuse is applied [10].

\subsection{Average Transmission Delay (ATD)}

The average number of slots between the slot when a packet is started to be transmitted by the node within certain level and the slot when it arrives at the BS.

\subsection{Slot Assignment Matrix (SAM)}

The routing algorithm significantly affects the scheduling algorithm while the Routing Tree (RT) is used as an input to the scheduling algorithm. An efficient RT can reduce interferences among the links, allows more spatial reuse and (consequently ) makes the scheduling algorithm capable to schedule more concurrent links over a specified slot. Therefore for each set $(10,20$.....100) of nodes used in the simulation, a SAM which is based on MFRCS algorithm is analyzed for each routing algorithm. The acquired parameters from the SAMs are used with the previous analytical equations to confirm the simulation results. The SAM must take into account the Uplink Interference Matrix (ULIM) for each routing algorithm and the Connectivity Matrix (CM), as follows :-

\section{a. Connectivity Matrix}

Connection link is existed between any two neighbors if the distance between them is less than their transmission range (TR). Refer to the mesh topology shown in Fig. (5), with flat (not arrowed) solid lines indicate the existence of connections between nodes, a CM $(n \times n)$ can be assigned, with the element $\left(n_{i}, n_{j}\right)$ set to 1 if $n_{i}$ is in the TR of $n_{j}$, as shown bellow in Fig. (10).

\begin{tabular}{|l|l|l|l|l|l|l|l|l|l|l|l|l|}
\hline & $\mathbf{0}$ & $\mathbf{1}$ & $\mathbf{2}$ & $\mathbf{3}$ & $\mathbf{4}$ & $\mathbf{5}$ & $\mathbf{6}$ & $\mathbf{7}$ & $\mathbf{8}$ & $\mathbf{9}$ & $\mathbf{1 0}$ & $\mathbf{1 1}$ \\
\hline $\mathbf{0}$ & & $\mathbf{1}$ & $\mathbf{1}$ & $\mathbf{1}$ & & & & & & & & \\
\hline $\mathbf{1}$ & $\mathbf{1}$ & & $\mathbf{1}$ & & $\mathbf{1}$ & & & & & & & \\
\hline $\mathbf{2}$ & $\mathbf{1}$ & $\mathbf{1}$ & & $\mathbf{1}$ & $\mathbf{1}$ & $\mathbf{1}$ & $\mathbf{1}$ & & & & & \\
\hline $\mathbf{3}$ & $\mathbf{1}$ & & $\mathbf{1}$ & & & & $\mathbf{1}$ & $\mathbf{1}$ & $\mathbf{1}$ & & & \\
\hline $\mathbf{4}$ & & $\mathbf{1}$ & $\mathbf{1}$ & & & $\mathbf{1}$ & & & & $\mathbf{1}$ & $\mathbf{1}$ & \\
\hline $\mathbf{5}$ & & & $\mathbf{1}$ & & $\mathbf{1}$ & & $\mathbf{1}$ & & & & $\mathbf{1}$ & \\
\hline $\mathbf{6}$ & & & $\mathbf{1}$ & $\mathbf{1}$ & & $\mathbf{1}$ & & $\mathbf{1}$ & & & & $\mathbf{1}$ \\
\hline 7 & & & & 1 & & & $\mathbf{1}$ & & $\mathbf{1}$ & & & $\mathbf{1}$ \\
\hline $\mathbf{8}$ & & & & $\mathbf{1}$ & & & & $\mathbf{1}$ & & & & $\mathbf{1}$ \\
\hline $\mathbf{9}$ & & & & & $\mathbf{1}$ & & & & & & $\mathbf{1}$ & \\
\hline $\mathbf{1 0}$ & & & & & $\mathbf{1}$ & $\mathbf{1}$ & & & & & $\mathbf{1}$ & \\
\hline 11 & & & & & & $\mathbf{1}$ & $\mathbf{1}$ & $\mathbf{1}$ & & & & \\
\hline \hline
\end{tabular}

Fig. (10) : CM for the mesh topology in Fig. (5) 


\section{b. Uplink Interference Matrix}

The ULIM is constructed based on the interference model given in (3.2) and the routing tree in (4). The ULIM is a (n-1) $\times(\mathrm{n}-1)$ matrix. If link $e_{i}$ interfere with link $e_{j}$ in the routing tree, the value of the element $\left(e_{i}, e_{j}\right)$ is either set $\mathrm{P}$ for primary interference or $\mathrm{S}$ for secondary interference. If the links are not interfered with each other, the element is set 1 and Fig. (11) illustrates the ULIM with and without directivity for the proposed and BFT-RTC algorithms.

\begin{tabular}{|c|c|c|c|c|c|c|c|c|c|c|c||}
\hline & $\mathbf{1}$ & $\mathbf{2}$ & $\mathbf{3}$ & $\mathbf{4}$ & $\mathbf{5}$ & $\mathbf{6}$ & $\mathbf{7}$ & $\mathbf{8}$ & $\mathbf{9}$ & $\mathbf{1 0}$ & $\mathbf{1 1}$ \\
\hline $\mathbf{1}$ & $\mathbf{1}$ & $\mathbf{P}$ & $\mathbf{P}$ & $\mathbf{S}$ & $\mathbf{S}$ & $\mathbf{S}$ & $\mathbf{1}$ & $\mathbf{1}$ & $\mathbf{S}$ & $\mathbf{S}$ & $\mathbf{1}$ \\
\hline $\mathbf{2}$ & $\mathbf{P}$ & $\mathbf{1}$ & $\mathbf{P}$ & $\mathbf{P}$ & $\mathbf{P}$ & $\mathbf{P}$ & $\mathbf{S}$ & $\mathbf{S}$ & $\mathbf{S}$ & $\mathbf{S}$ & $\mathbf{S}$ \\
\hline $\mathbf{3}$ & $\mathbf{P}$ & $\mathbf{P}$ & $\mathbf{1}$ & $\mathbf{S}$ & $\mathbf{S}$ & $\mathbf{S}$ & $\mathbf{P}$ & $\mathbf{P}$ & $\mathbf{1}$ & $\mathbf{1}$ & $\mathbf{S}$ \\
\hline $\mathbf{4}$ & $\mathbf{S}$ & $\mathbf{P}$ & $\mathbf{S}$ & $\mathbf{1}$ & $\mathbf{P}$ & $\mathbf{P}$ & $\mathbf{1}$ & $\mathbf{1}$ & $\mathbf{P}$ & $\mathbf{P}$ & $\mathbf{1}$ \\
\hline $\mathbf{5}$ & $\mathbf{S}$ & $\mathbf{P}$ & $\mathbf{S}$ & $\mathbf{P}$ & $\mathbf{1}$ & $\mathbf{P}$ & $\mathbf{1}$ & $\mathbf{1}$ & $\mathbf{S}$ & $\mathbf{S}$ & $\mathbf{S}$ \\
\hline $\mathbf{6}$ & $\mathbf{S}$ & $\mathbf{P}$ & $\mathbf{S}$ & $\mathbf{P}$ & $\mathbf{P}$ & $\mathbf{1}$ & $\mathbf{S}$ & $\mathbf{S}$ & $\mathbf{1}$ & $\mathbf{1}$ & $\mathbf{P}$ \\
\hline $\mathbf{7}$ & $\mathbf{1}$ & $\mathbf{S}$ & $\mathbf{P}$ & $\mathbf{1}$ & $\mathbf{1}$ & $\mathbf{S}$ & $\mathbf{1}$ & $\mathbf{P}$ & $\mathbf{1}$ & $\mathbf{1}$ & $\mathbf{S}$ \\
\hline $\mathbf{8}$ & $\mathbf{1}$ & $\mathbf{S}$ & $\mathbf{P}$ & $\mathbf{1}$ & $\mathbf{1}$ & $\mathbf{S}$ & $\mathbf{P}$ & $\mathbf{1}$ & $\mathbf{1}$ & $\mathbf{1}$ & $\mathbf{1}$ \\
\hline $\mathbf{9}$ & $\mathbf{S}$ & $\mathbf{S}$ & $\mathbf{1}$ & $\mathbf{P}$ & $\mathbf{S}$ & $\mathbf{1}$ & $\mathbf{1}$ & $\mathbf{1}$ & $\mathbf{1}$ & $\mathbf{P}$ & $\mathbf{1}$ \\
\hline $\mathbf{1 0}$ & $\mathbf{S}$ & $\mathbf{S}$ & $\mathbf{1}$ & $\mathbf{P}$ & $\mathbf{S}$ & $\mathbf{1}$ & $\mathbf{1}$ & $\mathbf{1}$ & $\mathbf{P}$ & $\mathbf{1}$ & $\mathbf{1}$ \\
\hline 11 & $\mathbf{1}$ & $\mathbf{S}$ & $\mathbf{S}$ & $\mathbf{1}$ & $\mathbf{S}$ & $\mathbf{P}$ & $\mathbf{S}$ & $\mathbf{1}$ & $\mathbf{1}$ & $\mathbf{1}$ & $\mathbf{1}$ \\
\hline
\end{tabular}

(a)

\begin{tabular}{||c|c|c|c|c|c|c|c|c|c|c|c||}
\hline & $\mathbf{1}$ & $\mathbf{2}$ & $\mathbf{3}$ & $\mathbf{4}$ & $\mathbf{5}$ & $\mathbf{6}$ & $\mathbf{7}$ & $\mathbf{8}$ & $\mathbf{9}$ & $\mathbf{1 0}$ & $\mathbf{1 1}$ \\
\hline $\mathbf{1}$ & $\mathbf{1}$ & $\mathbf{P}$ & $\mathbf{P}$ & $\mathbf{P}$ & $\mathbf{S}$ & $\mathbf{1}$ & $\mathbf{1}$ & $\mathbf{1}$ & $\mathbf{S}$ & $\mathbf{1}$ & $\mathbf{1}$ \\
\hline $\mathbf{2}$ & $\mathbf{P}$ & $\mathbf{1}$ & $\mathbf{P}$ & $\mathbf{S}$ & $\mathbf{P}$ & $\mathbf{S}$ & $\mathbf{S}$ & $\mathbf{S}$ & $\mathbf{S}$ & $\mathbf{S}$ & $\mathbf{1}$ \\
\hline $\mathbf{3}$ & $\mathbf{P}$ & $\mathbf{P}$ & $\mathbf{1}$ & $\mathbf{1}$ & $\mathbf{S}$ & $\mathbf{P}$ & $\mathbf{P}$ & $\mathbf{P}$ & $\mathbf{1}$ & $\mathbf{1}$ & $\mathbf{S}$ \\
\hline $\mathbf{4}$ & $\mathbf{P}$ & $\mathbf{S}$ & $\mathbf{1}$ & $\mathbf{1}$ & $\mathbf{S}$ & $\mathbf{1}$ & $\mathbf{1}$ & $\mathbf{1}$ & $\mathbf{P}$ & $\mathbf{S}$ & $\mathbf{1}$ \\
\hline $\mathbf{5}$ & $\mathbf{S}$ & $\mathbf{P}$ & $\mathbf{S}$ & $\mathbf{S}$ & $\mathbf{1}$ & $\mathbf{S}$ & $\mathbf{1}$ & $\mathbf{1}$ & $\mathbf{S}$ & $\mathbf{P}$ & $\mathbf{1}$ \\
\hline $\mathbf{6}$ & $\mathbf{1}$ & $\mathbf{S}$ & $\mathbf{P}$ & $\mathbf{1}$ & $\mathbf{S}$ & $\mathbf{1}$ & $\mathbf{P}$ & $\mathbf{P}$ & $\mathbf{1}$ & $\mathbf{S}$ & $\mathbf{S}$ \\
\hline $\mathbf{7}$ & $\mathbf{1}$ & $\mathbf{S}$ & $\mathbf{P}$ & $\mathbf{1}$ & $\mathbf{1}$ & $\mathbf{P}$ & $\mathbf{1}$ & $\mathbf{P}$ & $\mathbf{1}$ & $\mathbf{1}$ & $\mathbf{P}$ \\
\hline $\mathbf{8}$ & $\mathbf{1}$ & $\mathbf{S}$ & $\mathbf{P}$ & $\mathbf{1}$ & $\mathbf{1}$ & $\mathbf{P}$ & $\mathbf{P}$ & $\mathbf{1}$ & $\mathbf{1}$ & $\mathbf{1}$ & $\mathbf{S}$ \\
\hline $\mathbf{9}$ & $\mathbf{S}$ & $\mathbf{S}$ & $\mathbf{1}$ & $\mathbf{P}$ & $\mathbf{S}$ & $\mathbf{1}$ & $\mathbf{1}$ & $\mathbf{1}$ & $\mathbf{1}$ & $\mathbf{S}$ & $\mathbf{1}$ \\
\hline $\mathbf{1 0}$ & $\mathbf{1}$ & $\mathbf{S}$ & $\mathbf{1}$ & $\mathbf{S}$ & $\mathbf{P}$ & $\mathbf{S}$ & $\mathbf{1}$ & $\mathbf{1}$ & $\mathbf{S}$ & $\mathbf{1}$ & $\mathbf{S}$ \\
\hline $\mathbf{1 1}$ & $\mathbf{1}$ & $\mathbf{1}$ & $\mathbf{S}$ & $\mathbf{1}$ & $\mathbf{1}$ & $\mathbf{S}$ & $\mathbf{P}$ & $\mathbf{S}$ & $\mathbf{1}$ & $\mathbf{S}$ & $\mathbf{1}$ \\
\hline
\end{tabular}

(b)

\begin{tabular}{||c|c|c|c|c|c|c|c|c|c|c|c||}
\hline & $\mathbf{1}$ & $\mathbf{2}$ & $\mathbf{3}$ & $\mathbf{4}$ & $\mathbf{5}$ & $\mathbf{6}$ & $\mathbf{7}$ & $\mathbf{8}$ & $\mathbf{9}$ & $\mathbf{1 0}$ & $\mathbf{1 1}$ \\
\hline $\mathbf{1}$ & $\mathbf{1}$ & $\mathbf{P}$ & $\mathbf{P}$ & $\mathbf{P}$ & $\mathbf{S}$ & $\mathbf{S}$ & $\mathbf{1}$ & $\mathbf{1}$ & $\mathbf{S}$ & $\mathbf{1}$ & $\mathbf{1}$ \\
\hline $\mathbf{2}$ & $\mathbf{P}$ & $\mathbf{1}$ & $\mathbf{P}$ & $\mathbf{S}$ & $\mathbf{P}$ & $\mathbf{P}$ & $\mathbf{S}$ & $\mathbf{S}$ & $\mathbf{S}$ & $\mathbf{S}$ & $\mathbf{1}$ \\
\hline $\mathbf{3}$ & $\mathbf{P}$ & $\mathbf{P}$ & $\mathbf{1}$ & $\mathbf{1}$ & $\mathbf{S}$ & $\mathbf{S}$ & $\mathbf{P}$ & $\mathbf{P}$ & $\mathbf{1}$ & $\mathbf{1}$ & $\mathbf{S}$ \\
\hline $\mathbf{4}$ & $\mathbf{P}$ & $\mathbf{S}$ & $\mathbf{1}$ & $\mathbf{1}$ & $\mathbf{S}$ & $\mathbf{S}$ & $\mathbf{1}$ & $\mathbf{1}$ & $\mathbf{P}$ & $\mathbf{S}$ & $\mathbf{1}$ \\
\hline $\mathbf{5}$ & $\mathbf{S}$ & $\mathbf{P}$ & $\mathbf{S}$ & $\mathbf{S}$ & $\mathbf{1}$ & $\mathbf{P}$ & $\mathbf{1}$ & $\mathbf{1}$ & $\mathbf{S}$ & $\mathbf{P}$ & $\mathbf{1}$ \\
\hline $\mathbf{6}$ & $\mathbf{S}$ & $\mathbf{P}$ & $\mathbf{S}$ & $\mathbf{S}$ & $\mathbf{P}$ & $\mathbf{1}$ & $\mathbf{S}$ & $\mathbf{S}$ & $\mathbf{1}$ & $\mathbf{S}$ & $\mathbf{S}$ \\
\hline 7 & $\mathbf{1}$ & $\mathbf{S}$ & $\mathbf{P}$ & $\mathbf{1}$ & $\mathbf{1}$ & $\mathbf{S}$ & $\mathbf{1}$ & $\mathbf{P}$ & $\mathbf{1}$ & $\mathbf{1}$ & $\mathbf{P}$ \\
\hline $\mathbf{8}$ & $\mathbf{1}$ & $\mathbf{S}$ & $\mathbf{P}$ & $\mathbf{1}$ & $\mathbf{1}$ & $\mathbf{S}$ & $\mathbf{P}$ & $\mathbf{1}$ & $\mathbf{1}$ & $\mathbf{1}$ & $\mathbf{S}$ \\
\hline $\mathbf{9}$ & $\mathbf{S}$ & $\mathbf{S}$ & $\mathbf{1}$ & $\mathbf{P}$ & $\mathbf{S}$ & $\mathbf{1}$ & $\mathbf{1}$ & $\mathbf{1}$ & $\mathbf{1}$ & $\mathbf{S}$ & $\mathbf{1}$ \\
\hline $\mathbf{1 0}$ & $\mathbf{1}$ & $\mathbf{S}$ & $\mathbf{1}$ & $\mathbf{S}$ & $\mathbf{P}$ & $\mathbf{S}$ & $\mathbf{1}$ & $\mathbf{1}$ & $\mathbf{S}$ & $\mathbf{1}$ & $\mathbf{S}$ \\
\hline $\mathbf{1 1}$ & $\mathbf{1}$ & $\mathbf{1}$ & $\mathbf{S}$ & $\mathbf{1}$ & $\mathbf{1}$ & $\mathbf{S}$ & $\mathbf{P}$ & $\mathbf{S}$ & $\mathbf{1}$ & $\mathbf{S}$ & $\mathbf{1}$ \\
\hline
\end{tabular}

(c)

\begin{tabular}{||c|c|c|c|c|c|c|c|c|c|c|c||}
\hline & $\mathbf{1}$ & $\mathbf{2}$ & $\mathbf{3}$ & $\mathbf{4}$ & $\mathbf{5}$ & $\mathbf{6}$ & $\mathbf{7}$ & $\mathbf{8}$ & $\mathbf{9}$ & $\mathbf{1 0}$ & $\mathbf{1 1}$ \\
\hline $\mathbf{1}$ & $\mathbf{1}$ & $\mathbf{P}$ & $\mathbf{P}$ & $\mathbf{1}$ & $\mathbf{1}$ & $\mathbf{1}$ & $\mathbf{1}$ & $\mathbf{1}$ & $\mathbf{1}$ & $\mathbf{1}$ & $\mathbf{1}$ \\
\hline $\mathbf{2}$ & $\mathbf{P}$ & $\mathbf{1}$ & $\mathbf{P}$ & $\mathbf{P}$ & $\mathbf{P}$ & $\mathbf{P}$ & $\mathbf{1}$ & $\mathbf{1}$ & $\mathbf{1}$ & $\mathbf{1}$ & $\mathbf{1}$ \\
\hline $\mathbf{3}$ & $\mathbf{P}$ & $\mathbf{P}$ & $\mathbf{1}$ & $\mathbf{1}$ & $\mathbf{1}$ & $\mathbf{1}$ & $\mathbf{P}$ & $\mathbf{P}$ & $\mathbf{1}$ & $\mathbf{1}$ & $\mathbf{1}$ \\
\hline $\mathbf{4}$ & $\mathbf{1}$ & $\mathbf{P}$ & $\mathbf{1}$ & $\mathbf{1}$ & $\mathbf{P}$ & $\mathbf{P}$ & $\mathbf{1}$ & $\mathbf{1}$ & $\mathbf{P}$ & $\mathbf{P}$ & $\mathbf{1}$ \\
\hline $\mathbf{5}$ & $\mathbf{1}$ & $\mathbf{P}$ & $\mathbf{1}$ & $\mathbf{P}$ & $\mathbf{1}$ & $\mathbf{P}$ & $\mathbf{1}$ & $\mathbf{1}$ & $\mathbf{1}$ & $\mathbf{1}$ & $\mathbf{1}$ \\
\hline $\mathbf{6}$ & $\mathbf{1}$ & $\mathbf{P}$ & $\mathbf{1}$ & $\mathbf{P}$ & $\mathbf{P}$ & $\mathbf{1}$ & $\mathbf{1}$ & $\mathbf{1}$ & $\mathbf{1}$ & $\mathbf{1}$ & $\mathbf{P}$ \\
\hline $\mathbf{7}$ & $\mathbf{1}$ & $\mathbf{1}$ & $\mathbf{P}$ & $\mathbf{1}$ & $\mathbf{1}$ & $\mathbf{1}$ & $\mathbf{1}$ & $\mathbf{P}$ & $\mathbf{1}$ & $\mathbf{1}$ & $\mathbf{1}$ \\
\hline $\mathbf{8}$ & $\mathbf{1}$ & $\mathbf{1}$ & $\mathbf{P}$ & $\mathbf{1}$ & $\mathbf{1}$ & $\mathbf{1}$ & $\mathbf{P}$ & $\mathbf{1}$ & $\mathbf{1}$ & $\mathbf{1}$ & $\mathbf{1}$ \\
\hline $\mathbf{9}$ & $\mathbf{1}$ & $\mathbf{1}$ & $\mathbf{1}$ & $\mathbf{P}$ & $\mathbf{1}$ & $\mathbf{1}$ & $\mathbf{1}$ & $\mathbf{1}$ & $\mathbf{1}$ & $\mathbf{P}$ & $\mathbf{1}$ \\
\hline $\mathbf{1 0}$ & $\mathbf{1}$ & $\mathbf{1}$ & $\mathbf{1}$ & $\mathbf{P}$ & $\mathbf{1}$ & $\mathbf{1}$ & $\mathbf{1}$ & $\mathbf{1}$ & $\mathbf{P}$ & $\mathbf{1}$ & $\mathbf{1}$ \\
\hline $\mathbf{1 1}$ & $\mathbf{1}$ & $\mathbf{1}$ & $\mathbf{1}$ & $\mathbf{1}$ & $\mathbf{1}$ & $\mathbf{P}$ & $\mathbf{1}$ & $\mathbf{1}$ & $\mathbf{1}$ & $\mathbf{1}$ & $\mathbf{1}$ \\
\hline
\end{tabular}

(d)

\begin{tabular}{||c|c|c|c|c|c|c|c|c|c|c|c||}
\hline & $\mathbf{1}$ & $\mathbf{2}$ & $\mathbf{3}$ & $\mathbf{4}$ & $\mathbf{5}$ & $\mathbf{6}$ & $\mathbf{7}$ & $\mathbf{8}$ & $\mathbf{9}$ & $\mathbf{1 0}$ & $\mathbf{1 1}$ \\
\hline $\mathbf{1}$ & $\mathbf{1}$ & $\mathbf{P}$ & $\mathbf{P}$ & $\mathbf{P}$ & $\mathbf{1}$ & $\mathbf{1}$ & $\mathbf{1}$ & $\mathbf{1}$ & $\mathbf{1}$ & $\mathbf{1}$ & $\mathbf{1}$ \\
\hline $\mathbf{2}$ & $\mathbf{P}$ & $\mathbf{1}$ & $\mathbf{P}$ & $\mathbf{1}$ & $\mathbf{P}$ & $\mathbf{1}$ & $\mathbf{1}$ & $\mathbf{1}$ & $\mathbf{1}$ & $\mathbf{1}$ & $\mathbf{1}$ \\
\hline $\mathbf{3}$ & $\mathbf{P}$ & $\mathbf{P}$ & $\mathbf{1}$ & $\mathbf{1}$ & $\mathbf{1}$ & $\mathbf{P}$ & $\mathbf{P}$ & $\mathbf{P}$ & $\mathbf{1}$ & $\mathbf{1}$ & $\mathbf{1}$ \\
\hline $\mathbf{4}$ & $\mathbf{P}$ & $\mathbf{1}$ & $\mathbf{1}$ & $\mathbf{1}$ & $\mathbf{1}$ & $\mathbf{1}$ & $\mathbf{1}$ & $\mathbf{1}$ & $\mathbf{P}$ & $\mathbf{1}$ & $\mathbf{1}$ \\
\hline $\mathbf{5}$ & $\mathbf{1}$ & $\mathbf{P}$ & $\mathbf{1}$ & $\mathbf{1}$ & $\mathbf{1}$ & $\mathbf{1}$ & $\mathbf{1}$ & $\mathbf{1}$ & $\mathbf{1}$ & $\mathbf{P}$ & $\mathbf{1}$ \\
\hline $\mathbf{6}$ & $\mathbf{1}$ & $\mathbf{1}$ & $\mathbf{P}$ & $\mathbf{1}$ & $\mathbf{1}$ & $\mathbf{1}$ & $\mathbf{P}$ & $\mathbf{P}$ & $\mathbf{1}$ & $\mathbf{1}$ & $\mathbf{1}$ \\
\hline $\mathbf{7}$ & $\mathbf{1}$ & $\mathbf{1}$ & $\mathbf{P}$ & $\mathbf{1}$ & $\mathbf{1}$ & $\mathbf{P}$ & $\mathbf{1}$ & $\mathbf{P}$ & $\mathbf{1}$ & $\mathbf{1}$ & $\mathbf{P}$ \\
\hline $\mathbf{8}$ & $\mathbf{1}$ & $\mathbf{1}$ & $\mathbf{P}$ & $\mathbf{1}$ & $\mathbf{1}$ & $\mathbf{P}$ & $\mathbf{P}$ & $\mathbf{1}$ & $\mathbf{1}$ & $\mathbf{1}$ & $\mathbf{1}$ \\
\hline $\mathbf{9}$ & $\mathbf{1}$ & $\mathbf{1}$ & $\mathbf{1}$ & $\mathbf{P}$ & $\mathbf{1}$ & $\mathbf{1}$ & $\mathbf{1}$ & $\mathbf{1}$ & $\mathbf{1}$ & $\mathbf{1}$ & $\mathbf{1}$ \\
\hline 10 & $\mathbf{1}$ & $\mathbf{1}$ & $\mathbf{1}$ & $\mathbf{1}$ & $\mathbf{P}$ & $\mathbf{1}$ & $\mathbf{1}$ & $\mathbf{1}$ & $\mathbf{1}$ & $\mathbf{1}$ & $\mathbf{1}$ \\
\hline $\mathbf{1 1}$ & $\mathbf{1}$ & $\mathbf{1}$ & $\mathbf{1}$ & $\mathbf{1}$ & $\mathbf{1}$ & $\mathbf{1}$ & $\mathbf{P}$ & $\mathbf{1}$ & $\mathbf{1}$ & $\mathbf{1}$ & $\mathbf{1}$ \\
\hline \hline
\end{tabular}

(e)

\begin{tabular}{||c|c|c|c|c|c|c|c|c|c|c|c||}
\hline & $\mathbf{1}$ & $\mathbf{2}$ & $\mathbf{3}$ & $\mathbf{4}$ & $\mathbf{5}$ & $\mathbf{6}$ & $\mathbf{7}$ & $\mathbf{8}$ & $\mathbf{9}$ & $\mathbf{1 0}$ & $\mathbf{1 1}$ \\
\hline $\mathbf{1}$ & $\mathbf{1}$ & $\mathbf{P}$ & $\mathbf{P}$ & $\mathbf{P}$ & $\mathbf{1}$ & $\mathbf{1}$ & $\mathbf{1}$ & $\mathbf{1}$ & $\mathbf{1}$ & $\mathbf{1}$ & $\mathbf{1}$ \\
\hline $\mathbf{2}$ & $\mathbf{P}$ & $\mathbf{1}$ & $\mathbf{P}$ & $\mathbf{1}$ & $\mathbf{P}$ & $\mathbf{p}$ & $\mathbf{1}$ & $\mathbf{1}$ & $\mathbf{1}$ & $\mathbf{1}$ & $\mathbf{1}$ \\
\hline $\mathbf{3}$ & $\mathbf{P}$ & $\mathbf{P}$ & $\mathbf{1}$ & $\mathbf{1}$ & $\mathbf{1}$ & $\mathbf{1}$ & $\mathbf{P}$ & $\mathbf{P}$ & $\mathbf{1}$ & $\mathbf{1}$ & $\mathbf{1}$ \\
\hline $\mathbf{4}$ & $\mathbf{P}$ & $\mathbf{1}$ & $\mathbf{1}$ & $\mathbf{1}$ & $\mathbf{1}$ & $\mathbf{1}$ & $\mathbf{1}$ & $\mathbf{1}$ & $\mathbf{P}$ & $\mathbf{1}$ & $\mathbf{1}$ \\
\hline $\mathbf{5}$ & $\mathbf{1}$ & $\mathbf{P}$ & $\mathbf{1}$ & $\mathbf{1}$ & $\mathbf{1}$ & $\mathbf{P}$ & $\mathbf{1}$ & $\mathbf{1}$ & $\mathbf{1}$ & $\mathbf{P}$ & $\mathbf{1}$ \\
\hline $\mathbf{6}$ & $\mathbf{1}$ & $\mathbf{P}$ & $\mathbf{1}$ & $\mathbf{1}$ & $\mathbf{P}$ & $\mathbf{1}$ & $\mathbf{1}$ & $\mathbf{1}$ & $\mathbf{1}$ & $\mathbf{1}$ & $\mathbf{1}$ \\
\hline 7 & $\mathbf{1}$ & $\mathbf{1}$ & $\mathbf{P}$ & $\mathbf{1}$ & $\mathbf{1}$ & $\mathbf{1}$ & $\mathbf{1}$ & $\mathbf{P}$ & $\mathbf{1}$ & $\mathbf{1}$ & $\mathbf{P}$ \\
\hline $\mathbf{8}$ & $\mathbf{1}$ & $\mathbf{1}$ & $\mathbf{P}$ & $\mathbf{1}$ & $\mathbf{1}$ & $\mathbf{1}$ & $\mathbf{P}$ & $\mathbf{1}$ & $\mathbf{1}$ & $\mathbf{1}$ & $\mathbf{1}$ \\
\hline $\mathbf{9}$ & $\mathbf{1}$ & $\mathbf{1}$ & $\mathbf{1}$ & $\mathbf{P}$ & $\mathbf{1}$ & $\mathbf{1}$ & $\mathbf{1}$ & $\mathbf{1}$ & $\mathbf{1}$ & $\mathbf{1}$ & $\mathbf{1}$ \\
\hline $\mathbf{1 0}$ & $\mathbf{1}$ & $\mathbf{1}$ & $\mathbf{1}$ & $\mathbf{1}$ & $\mathbf{P}$ & $\mathbf{1}$ & $\mathbf{1}$ & $\mathbf{1}$ & $\mathbf{1}$ & $\mathbf{1}$ & $\mathbf{1}$ \\
\hline 11 & $\mathbf{1}$ & $\mathbf{1}$ & $\mathbf{1}$ & $\mathbf{1}$ & $\mathbf{1}$ & $\mathbf{P}$ & $\mathbf{1}$ & $\mathbf{1}$ & $\mathbf{1}$ & $\mathbf{1}$ & $\mathbf{1}$ \\
\hline
\end{tabular}

(f)

Fig. (11) shows the ULIM (a,b,c) without and (d,e,f) with directivity for BFT and Bala-Para RTCAs (without and with DNGT) 
The procedure of analyzing the SAM is based on the fact that the nearest node to the BS is scheduled for transmission first and more than one node can be scheduled at the same slot if they are not interfered with each other. So by referring to the ULIM, the SAMs are constructed for the standard and proposed RTC algorithms as shown in Fig. (12).
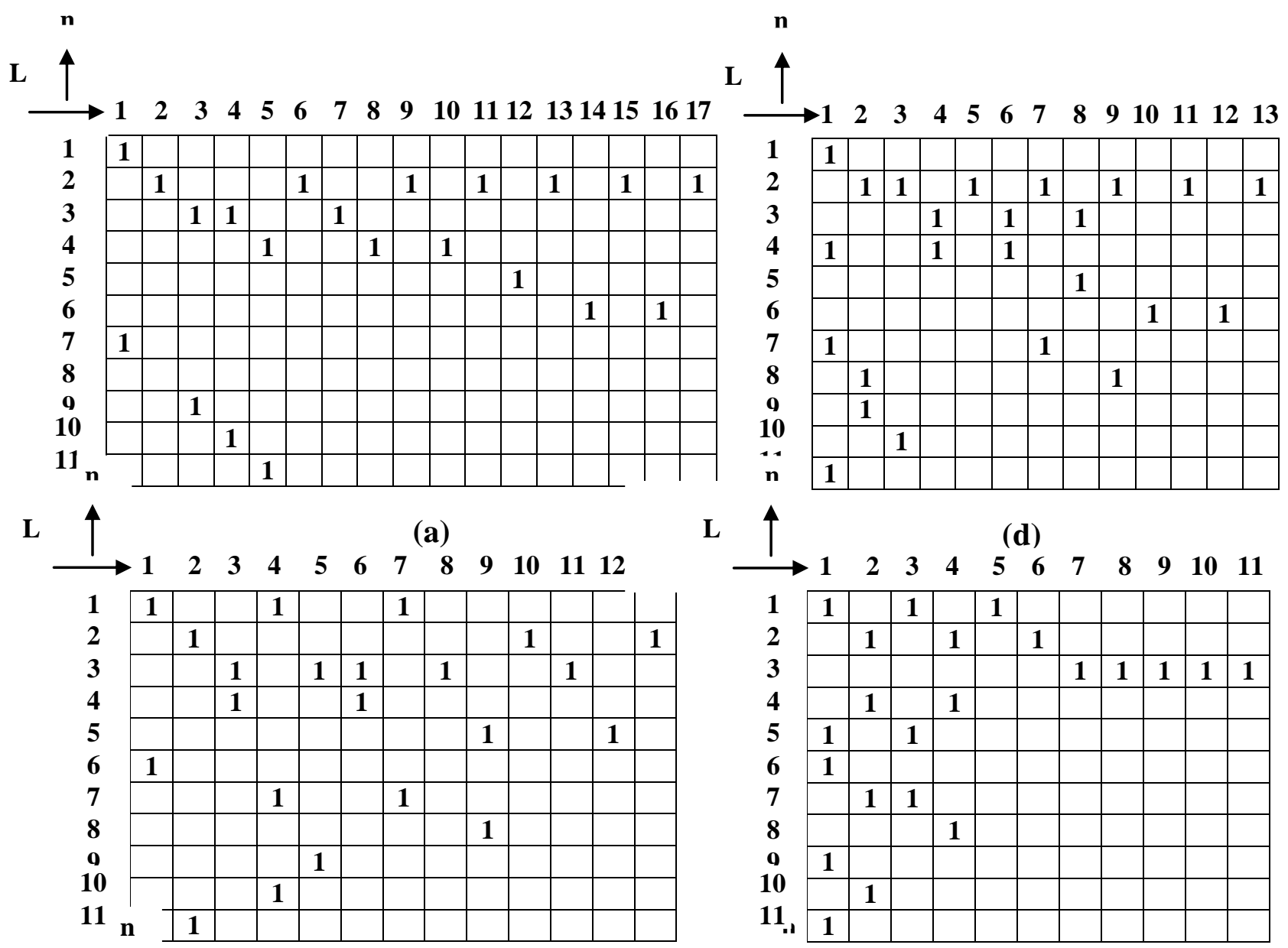

$\mathbf{L}$
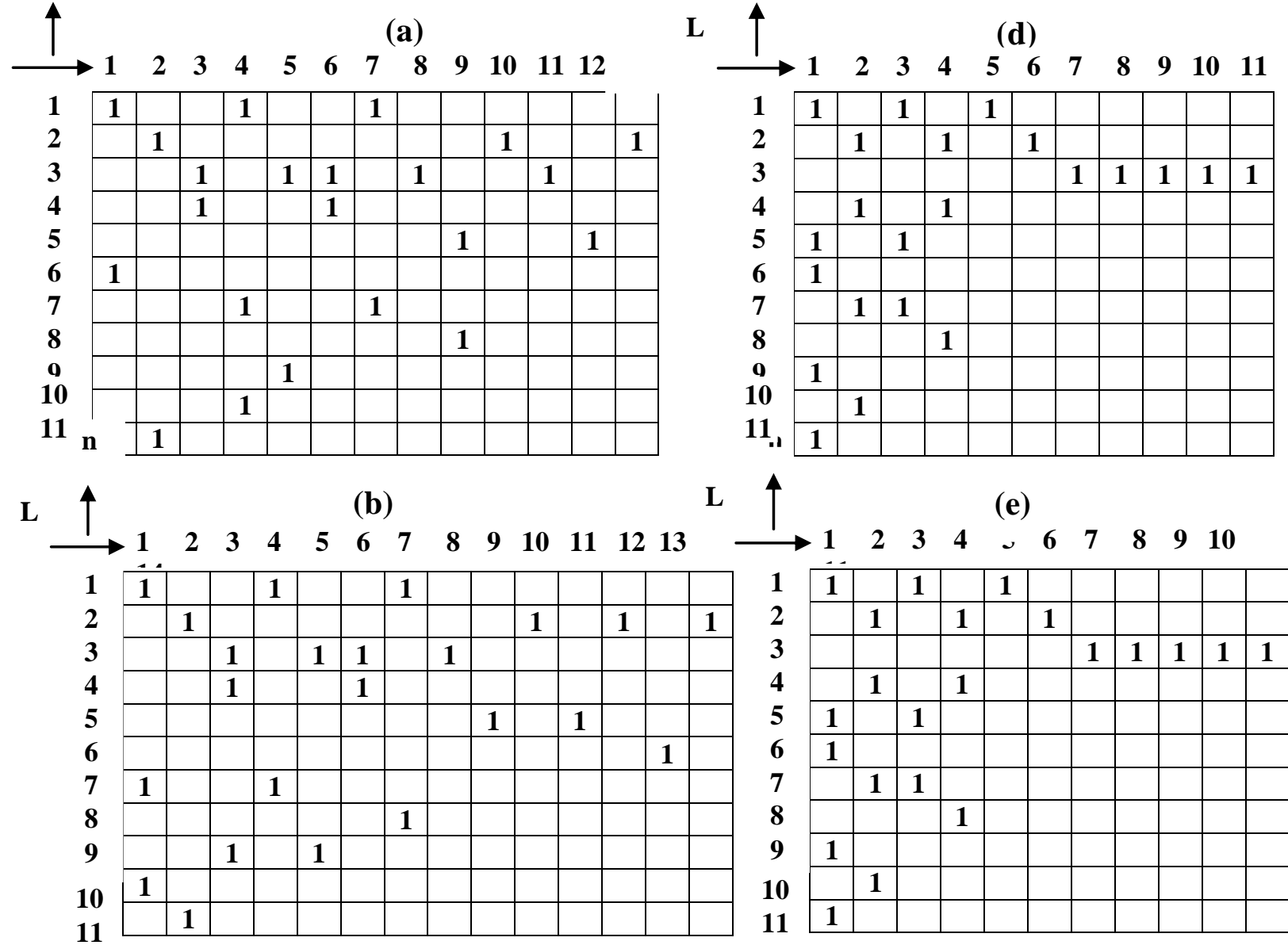

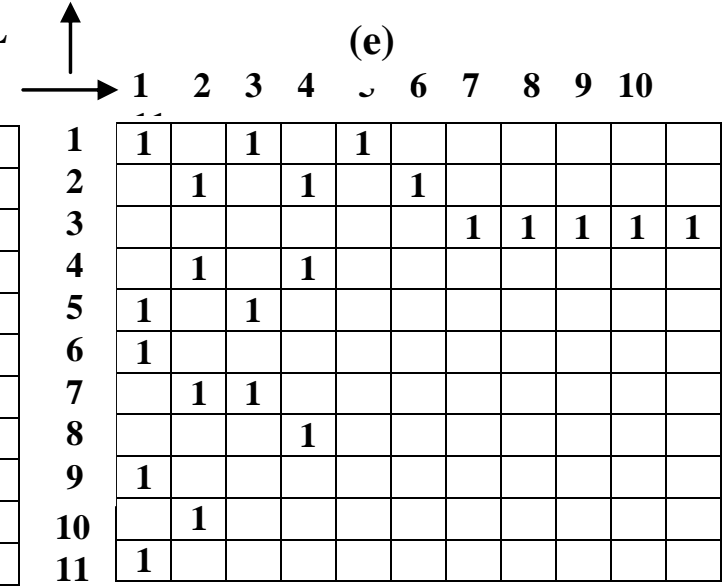

(c)

(f)

Fig. (12): slot assign (c) without and (d), (e), (f) with directivity for BFT, Bala-Para RTC algorithms (without and with DNGT) respectively 
From the SAM and as a matter of check, the following can be obtained:-

1) The ATD for the three routing algorithm is given as follow:-

We have unified traffic demand $\left(D_{i}=1\right)$, level $=3, \mathrm{n}=$ number of $\mathrm{SSs}=11$, the $\mathrm{SS}_{9}, \mathrm{SS}_{10}$ and $\mathrm{SS}_{11}$ are at level 3, if transmission slot (i) is denoted by $\mathrm{T}_{\mathrm{i}}$ then for;

a) BFT Routing Algorithm

i) Without directivity

Number of slots for packet to reach BS from $\mathrm{SS}_{9}=\mathrm{T}_{9}-\mathrm{T}_{3}=6$

Number of slots for packet to reach BS from $\mathrm{SS}_{10}=\mathrm{T}_{11}-\mathrm{T}_{4}=7$

Number of slots for packet to reach BS from $\mathrm{SS}_{11}=\mathrm{T}_{17}-\mathrm{T}_{5}=12$

$\therefore \mathrm{ATD}=\frac{6+7+12}{3}=\frac{25}{3}=8.33$ slots

ii) With directivity

Number of slots for packet to reach BS from $\mathrm{SS}_{9}=\mathrm{T}_{5}-\mathrm{T}_{2}=3$

Number of slots for packet to reach BS from $\mathrm{SS}_{10}=\mathrm{T}_{7}-\mathrm{T}_{3}=4$

Number of slots for packet to reach BS from $\mathrm{SS}_{11}=\mathrm{T}_{13}-\mathrm{T}_{1}=12$

$\therefore \mathrm{ATD}=\frac{3+4+12}{3}=\frac{19}{3}=6.33$ slots

b) Bala-Para-RTC Algorithm without DNGT feature

i) Without directivity

Number of slots for packet to reach BS from $\mathrm{SS}_{9}=\mathrm{T}_{7}-\mathrm{T}_{5}=2$

Number of slots for packet to reach BS from $\mathrm{SS}_{10}=\mathrm{T}_{13}-\mathrm{T}_{4}=9$

Number of slots for packet to reach BS from $\mathrm{SS}_{11}=\mathrm{T}_{8}-\mathrm{T}_{2}=6$

$\mathrm{ATD}=\frac{2+9+6}{3}=\frac{17}{3}=5.6$ slots

ii) With directivity

Number of slots for packet to reach BS from $\mathrm{SS}_{9}=\mathrm{T}_{5}-\mathrm{T}_{1}=4$

Number of slots for packet to reach BS from $\mathrm{SS}_{10}=\mathrm{T}_{6}-\mathrm{T}_{2}=4$

Number of slots for packet to reach BS from $\mathrm{SS}_{11}=\mathrm{T}_{10}-\mathrm{T}_{1}=9$

$\mathrm{ATD}=\frac{4+4+9}{3}=\frac{17}{3}=5.6$ slots

c) Bala-Para-RTC Algorithm with DNGT feature

i) Without directivity

Number of slots for packet to reach BS from $\mathrm{SS}_{9}=\mathrm{T}_{7}-\mathrm{T}_{5}=2$

Number of slots for packet to reach BS from $S_{10}=T_{12}-T_{1}=11$

Number of slots for packet to reach BS from $\mathrm{n}_{11}=\mathrm{T}_{6}-\mathrm{T}_{2}=4$

$\mathrm{ATD}=\frac{2+11+4}{3}=\frac{17}{3}=5.6$ slots

ii) With directivity

Number of slots for packet to reach BS from $\mathrm{n}_{9}=\mathrm{T}_{5}-\mathrm{T}_{1}=4$

Number of slots for packet to reach BS from $\mathrm{n}_{10}=\mathrm{T}_{6}-\mathrm{T}_{2}=4$

Number of slots for packet to reach BS from $\mathrm{n}_{11}=\mathrm{T}_{11}-\mathrm{T}_{2}=9$

$\mathrm{ATD}=\frac{4+4+9}{3}=\frac{17}{3}=5.6$ slots

2) The actual scheduling length (L) can be obtained directly from SAM

3) The occupied slots will represent the upper bound scheduling length when only one link is scheduled every slot and LCR can be calculated .

4) The CUR (given by equation (6)) can be calculated directly from SAM as follows:-

a. The occupied slots $=\sum_{i=1}^{n} \mathrm{~h}_{i} \mathrm{D}_{i}$

$$
=\sum_{i=1}^{n} \mathrm{~h}_{i} \text { for unified traffic }\left(\mathrm{D}_{i}=1\right)
$$


$=3 \mathrm{SS} \times 1$ st hop $+5 \mathrm{SS} \times 2$ nd hop $+3 \mathrm{SS} \times 3 \mathrm{rd}$ hop $=22$

b. Without directivity, the available (nL) slots are 187, 143 and 154 for BFT, the proposed Bala-Para RTC algorithms respectively.

c. With directivity, the available slots are 143,121 and 121 for BFT and the proposed algorithms respectively.

\section{Simulation results}

Qualnet simulator version 5.0.2 is used to evaluate the performance of the proposed (Para and Bala-Para) routing algorithms. The results are compared with the results of the standard BFT-RTCA.

The simulation results are based on the following assumptions :

a. The simulation area being used is a $100 \times 100$ units.

b. The BS is placed at the middle of the cell.

c. In the long term, all nodes have the same average traffic demand therefore, for unified traffic, it is assumed that all the stations generate the same amount of uplink traffic (1 packet each) and hence request the same amount of recourses (1 slot each).

d. One packet is transmitted in each slot and two nodes are neighbors only if they are in the transmission range of each other.

e. Power control is applied when directivity is used.

f. All nodes have the same transmission range $\mathrm{TR}=22.5$ units for maximum hoping range of five hops.

g. The SSs (ranges from 10 to 100 with increment step of 10) has been scattered randomly and uniformly in the topology of the cell.

h. The number of active links were varying randomly depending on the involved interferences among links.

i. To account for the randomness behavior of active links, readings were averaged over 10 connected random mesh topologies for each set $(10,20,30, \ldots . .100)$ of nodes involved in the topology.

j. Maximum hop range is five.

k. For directivity, every SS is equipped with six directional antennas, each one cover 60 degree, one of them is switched on (depending on direction of transmission or reception ) when SS is scheduled at certain slot.

\section{i. Length of schedule :}

The results of the relationship between the length of scheduling and the number of nodes are shown in Figures (13a,b,c,).

Figure (13) shows that for higher average traffic demand, the length of schedule increases more rapidly as the number of nodes increases. It is clear that the directivity produces maximum reductions in the schedule lengths (around 8, 10, and 12 slots) when the number of nodes are 40, 50 and 60 respectively.

Figures(14.a) and (15.a) show the relationship between the length of schedule and the number of nodes for the routing algorithm without and with directivity respectively. Due to the parallelism and balancing properties, the performance of the proposed routing algorithms to reduce the length of schedule is better than the BFT-RTCA. On the other hand, the performance without directivity (when the number of nodes=100 and as far as BFT-RTCA is concerned) shows that the Bala-Para RTCAs have schedule reductions of 18 and 20 slots, 
while with directivity they have a reduction of 20 and 22 slots without and with DNGT respectively.

For variable traffic demand with directivity (i.e spatial reuse is allowed more frequently), the feature (which gives priority to down node group) of the Bala-Para routing algorithm shows higher effect on the scheduling length and this can be shown from Figure (15.a) where the performance of Bala-Para DNGT is better than that without DNGT.

The lower and upper bounds values are almost given by the number of nodes multiplied by the average traffic demand values. The upper bound shows high values because only one link is scheduled in every slot.

ii.Link Concurrency Ratio (LCR): The results of the LCR as a function of the number of nodes are shown in Figures (13d,e,f)

Figure (13) shows that the LCR increases as the number of nodes increases. In general, without and with directivity, the proposed algorithm achieve better performance than BFT. The directivity shows better performance for the routing algorithms. However, the Bala-Para algorithm with DNGT has the maximum range (up to 80 nodes) over which directivity exists, while BFT and Bala-Para without DNGT algorithms have ranges which are up to 50 and 70 respectively. The obtained maximum values are 3.25, 3.75 and 3.88 for BFT, Bala-Para without and with DNGT respectively.

Figure (14.b) shows that the proposed algorithm achieve better performance than the BFTRTCA and the rate at which the LCR will be increased is higher than that of BFT. Without directivity the obtained maximum values are 3.1, 3.4 and 3.43 for BFT, Bala-Para without and with DNGT respectively.

Figure (15.b) shows that the range of nodes over which the LCR of the Bala-Para-RTCA with DNGT attains maximum values is higher than that without DNGT. The Figure also shows that with directivity the Bala-Para with DNGT achieves better performance than that without DNGT for high number of nodes $(>50)$ and the rate at which the LCR will be decreased is higher than that without DNGT. This is related to the priority given to the highest BW or ID nodes within the down node groups, this is required to choose the rout first and to make the algorithm more resistant to the interferences as the number of nodes exceeds the range 50-60. It is worth to mention that the faster the increasing rate of LCR, the lower the ATD. Also the higher concurrency ratio over wider range of nodes will lead to the usage of most of the available links over the network efficiently (i.e more scalable).

iii. Average Transmission Delay (ATD): For multihop networks, the ATD increases as the number of levels increases. Since it makes no sense to study the ATD over the whole net, the ATD of subscribers which are at level 3 from the BS is to be considered. The result of the average transmission delay as a function of the number of nodes of the different routing algorithms are shown in Figures (16) and (17). The Figures show that the ATD will be increased as the number of nodes increases, as an example, if the number of nodes $=100$, then maximum delays of 56, 52 and 50 for BFT, Bala-Para RTCA without and with DNGT respectively can be obtained.

For BFT-RTC algorithm, at very low $(<20)$ number of nodes, the directivity shows higher effect as far as the reduction of ATD is concerned.

Figure (17) shows that the ATD is more steeper beyond the nodes range (40-50). This means as the average traffic demand increases, the more and at lower range of nodes the ATD becomes steeper. The directivity has higher effect on the ATD when the number of nodes is less or equals to 40. The reason for that is related to the directivity which allows more 
concurrent transmissions for far nodes. Consequently, congestion and delay at intermediate nodes will be obtained.
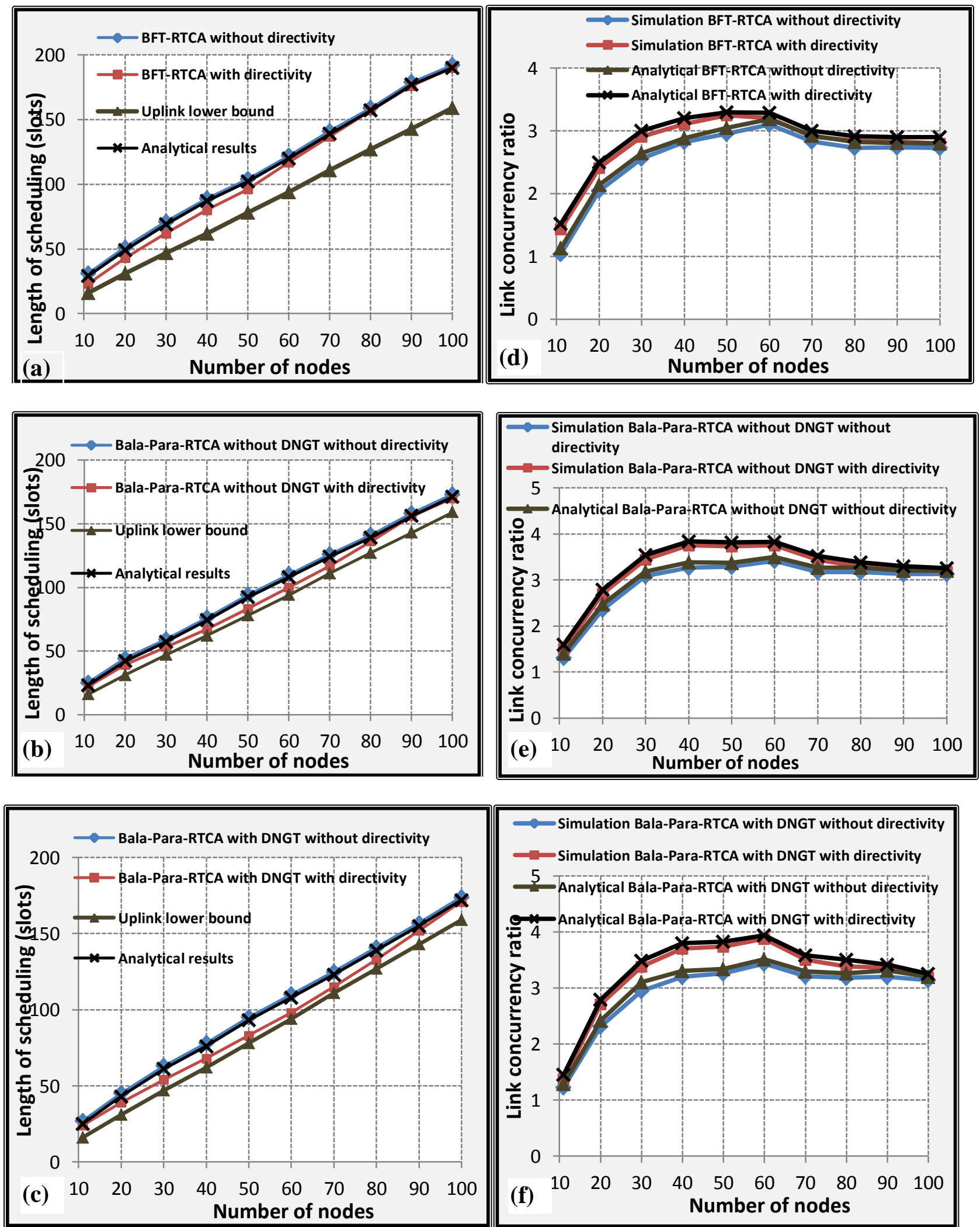

Fig. (13): shows the relationship between the length of schedule (a,b,c) and the link concurrency ratio (d,e,f) against the number of nodes for BFT, Bala-Para-RTCA (without and with DNGT) when the traffic demand is variable $(0-3)$ and $\mathrm{TR}=22.5$ 


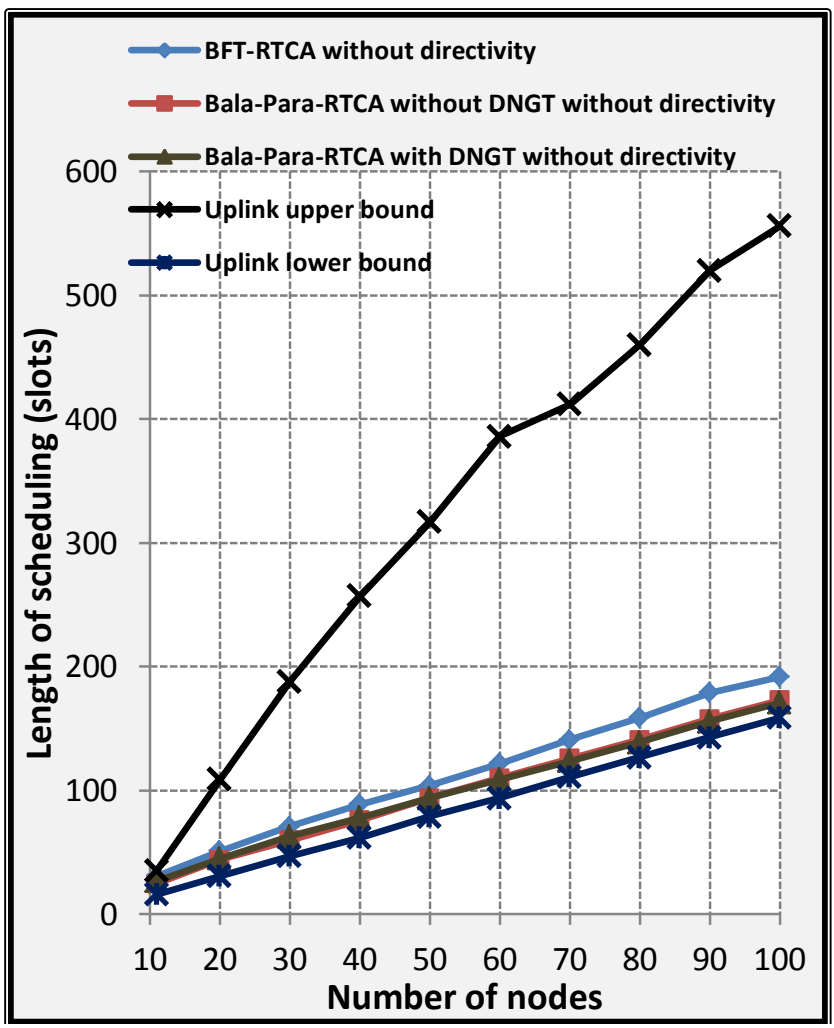

(a)

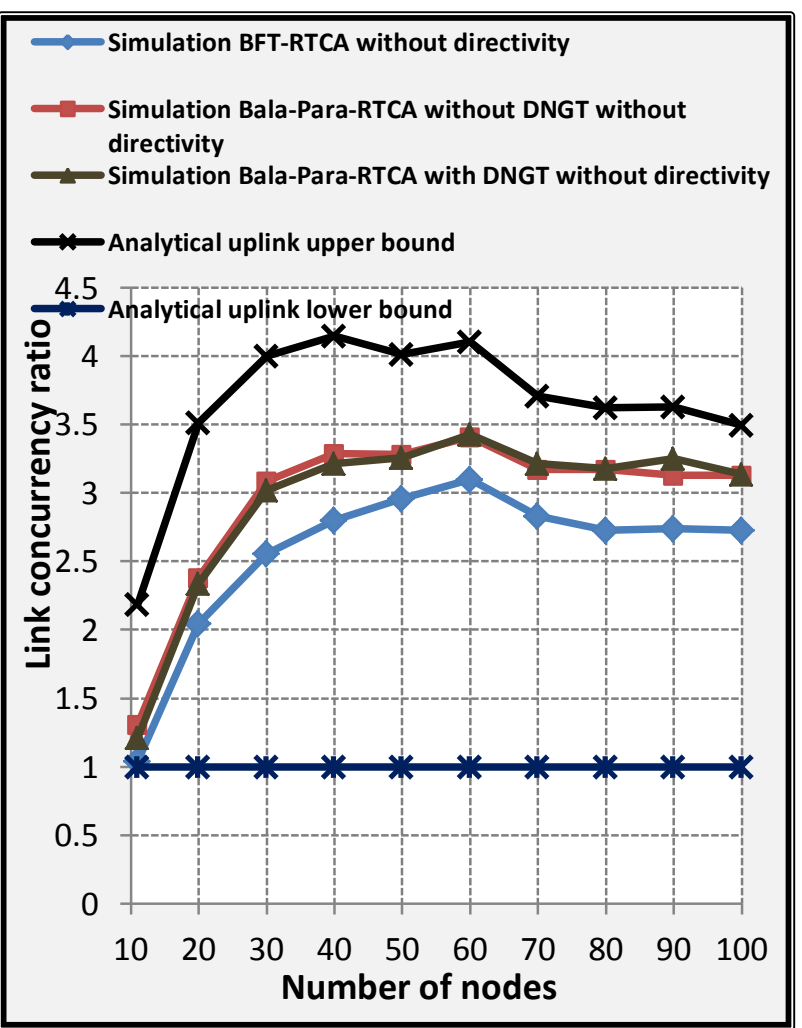

(b)

Fig. (14): shows the relationship between (a) the length of schedule and (b) link concurrency ratio against the number of nodes for BFT, Bala-Para-RTCA (without and with DNGT) when the traffic demand is variable $(0-3)$ and $\mathrm{TR}=22.5$
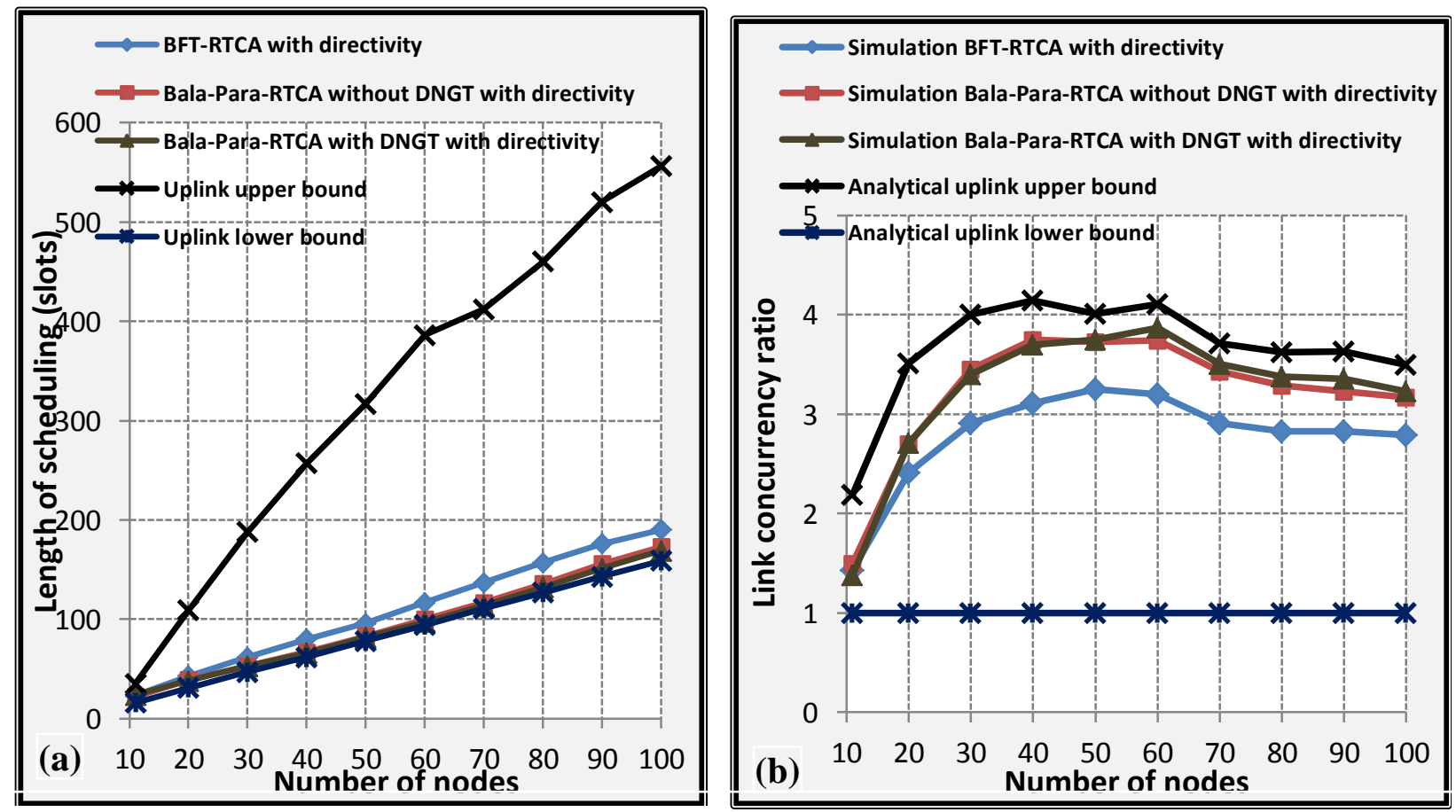

Fig. (15): shows the relationship between (a) the length of schedule and (b) link concurrency ratio against the number of nodes for BFT, Bala-Para-RTCA (without and with DNGT) when the traffic demand is variable $(0-3)$ and $\mathrm{TR}=22.5$ 

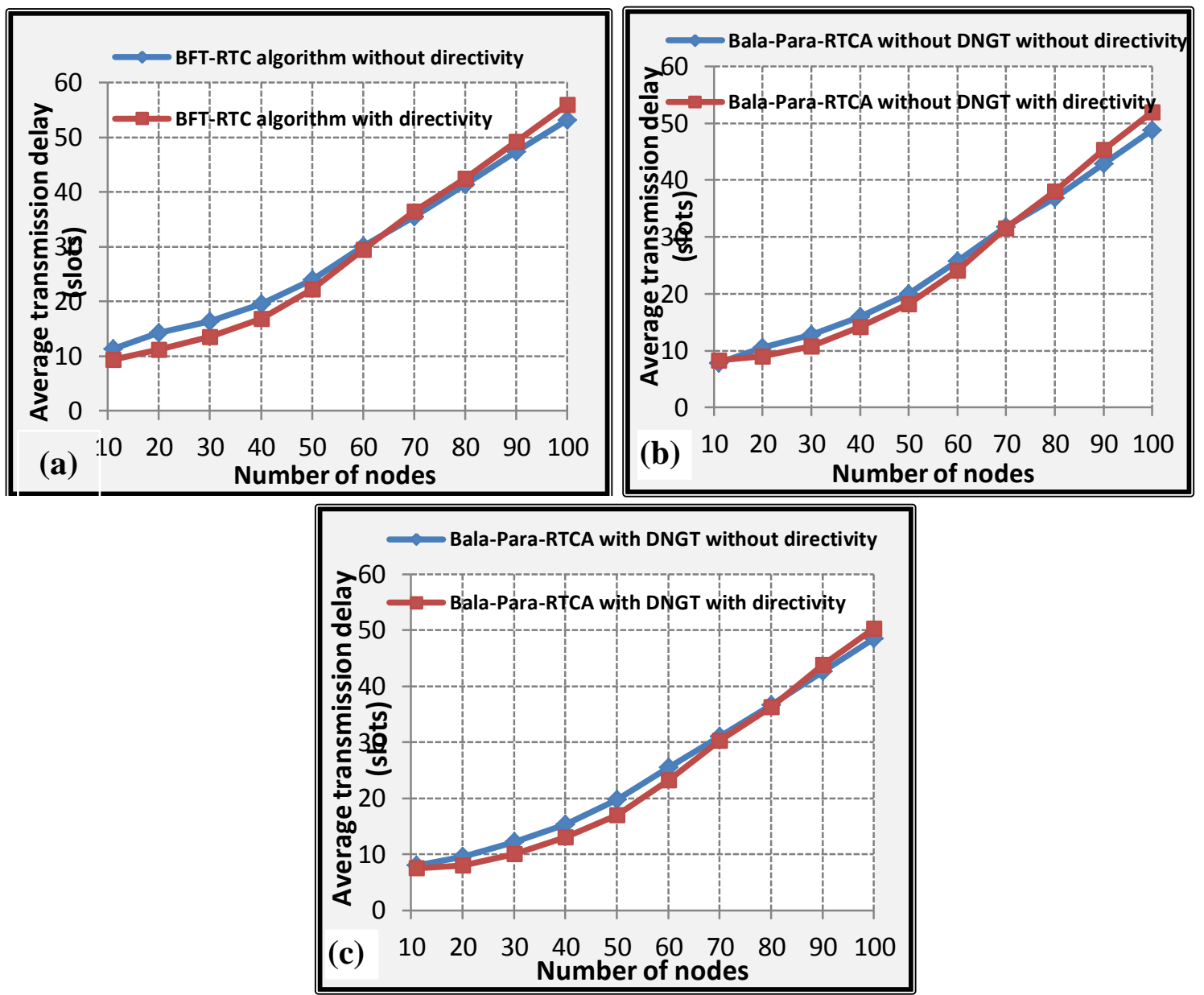

Fig. (16): shows the average transmission delay against the number of nodes for BFT, Bala-Para-RTCA (without and with DNGT) when the traffic demand is variable (0-3) and $\mathrm{TR}=22.5$
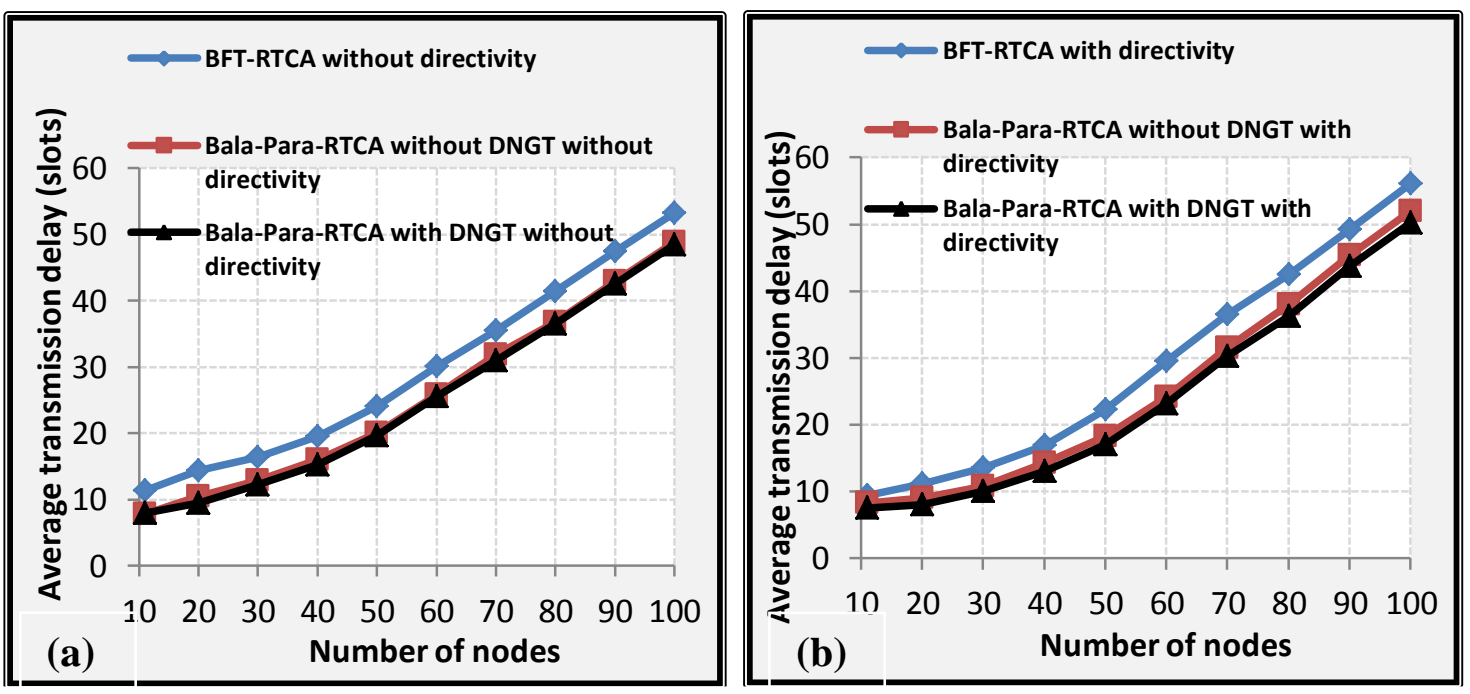

Fig. (17): shows the average transmission delay against the number of nodes for BFT, Bala-Para-RTCA (without and with DNGT) when the traffic demand is variable $(0-3)$ and $\mathrm{TR}=22.5$ 


\section{Conclusion}

The trend of the relay scheduling models is to impose fairness on WiMAX mesh network. In this paper, the proposed Bala-Para RTCA, which is based on parallelism \& balancing, with its new feature (DNGT) is used to enhance the system performance. With directivity, the proposed routing algorithm without and with DNGT provide a maximum reduction in the length of schedule of around 20 and 22 slots respectively, at a number of nodes $=100$. Also they show significant improvement in LCR of about $15.38 \% \& 19.4 \%$ relative to the conventional BFT algorithm.

For variable traffic demand, system directivity shows an improvement in LCR over large number (up to 80) of nodes and an acceptable reduction in the ATD up to nodes range (4050), therefore it is possible to conclude that the proposed routing algorithm with and without DNGT is suitable for large scale and medium dense networks.

\section{REFERENCES}

[1] IEEE Std 802.16-2004. IEEE Standard for Local and metropolitan area networks Part 16; air interface for fixed broadband wireless access systems, 2004.

[2] F. Jin, A. Arora, J. Hwang, and H. A. Choi, "Routing and Packet Scheduling for Throughput Maximization in IEEE 802.16 Mesh Networks," in Proc. IEEE Intl. Conf. on Broadband Networks, Sept. 2007.

[3] H. Y. Wei, S. Ganguly, and R. Izmailov, "Interference-Aware IEEE 802.16 WiMax Mesh Networks," in Proc. IEEE Vehicular Technology Conf. (VTC), vol. 5, pp.3102-3106, 2005.

[4] B. Han, W. Jia, and L. Lin, "Performance Evaluation of Scheduling in IEEE 802.16 Based Wireless Mesh Networks," in Proc. IEEE Intl. Conf. on Mobile Adhoc and Sensor Systems (MASS), pp. 789-794, Oct. 2006.

[5] J. Tao, F. Liu and Z. Zeng, " Throughput Enhancement in WiMax Mesh Networks Using Concurrent Transmission", in proc. IEEE International Conference on Wireless Communications, Networking and Mobile Computing, Pages: 871-874, 2005.

[6] Y. Lu and Guoqing, " Maintaining Routing Tree in IEEE 802.16 Centralized Scheduling Mesh Networks", International Conference on Computer Communications and Networks, Pages: 240-245, 2006.

[7] P. Du . J. Wang. W. Jia . L. Huang and H. Li, "Minimizing Transmission Time in 802.16-Based Multi-Channel Mesh Networks," International Journal of Pervasive Computing and Communications Vol. 5 No. 3, 2009 pp. 295-311.

[8] Y. Tang, Rung-S. Cheng, T. Wu, and Jian-Ping Yu, "On Centralized Scheduling and Channel Assignment Scheme for WiMAX Mesh Networks," Journal of Internet Technology Volume 10, No.3 2009.

[9] W. Jiao, P. Jiang and R. Liu, " Centralized Scheduling Tree Construction under Multichannel IEEE 802.16 Mesh Networks", in proc. IEEE GLOBECOM (Global Telecommunications) Conference, Pages: 4764-4768, 2007.

[10] S. Liu, S. Feng, W. Yea and H. Zhuang, " Slot allocation algorithms in centralized scheduling scheme for IEEE 802.16 based wireless mesh networks", Elsevier Journal of Computer Communications, pp. 943-953, 32 (2009).

The work was carried out at the college of Engineering. University of Mosul 